\title{
Review Article \\ Yoga for Adults with Type 2 Diabetes: A Systematic Review of Controlled Trials
}

\author{
Kim E. Innes ${ }^{1,2}$ and Terry Kit Selfe $e^{1,2}$ \\ ${ }^{1}$ Department of Epidemiology, West Virginia University School of Public Health, Morgantown, WV 26506, USA \\ ${ }^{2}$ Center for the Study of Complementary and Alternative Therapies, University of Virginia Health System, Charlottesville, \\ VA 22903, USA \\ Correspondence should be addressed to Kim E. Innes; kinnes@hsc.wvu.edu
}

Received 2 June 2015; Revised 19 August 2015; Accepted 26 August 2015

Academic Editor: Mitsuhiko Noda

Copyright ( $) 2016$ K. E. Innes and T. K. Selfe. This is an open access article distributed under the Creative Commons Attribution License, which permits unrestricted use, distribution, and reproduction in any medium, provided the original work is properly cited.

\begin{abstract}
A growing body of evidence suggests yogic practices may benefit adults with type 2 diabetes (DM2). In this systematic review, we evaluate available evidence from prospective controlled trials regarding the effects of yoga-based programs on specific health outcomes pertinent to DM2 management. To identify qualifying studies, we searched nine databases and scanned bibliographies of relevant review papers and all identified articles. Controlled trials that did not target adults with diabetes, included only adults with type 1 diabetes, were under two-week duration, or did not include quantitative outcome data were excluded. Study quality was evaluated using the PEDro scale. Thirty-three papers reporting findings from 25 controlled trials (13 nonrandomized, 12 randomized) met our inclusion criteria ( $N=2170$ participants). Collectively, findings suggest that yogic practices may promote significant improvements in several indices of importance in DM2 management, including glycemic control, lipid levels, and body composition. More limited data suggest that yoga may also lower oxidative stress and blood pressure; enhance pulmonary and autonomic function, mood, sleep, and quality of life; and reduce medication use in adults with DM2. However, given the methodological limitations of existing studies, additional high-quality investigations are required to confirm and further elucidate the potential benefits of yoga programs in populations with DM2.
\end{abstract}

\section{Introduction}

Type 2 diabetes (DM2) has become a leading public health issue globally, with estimated 366 million people affected in 2011 [1]. This figure represents a more than twofold rise in the last three decades and parallels the growing pandemic of obesity and the increasingly widespread adoption of Western lifestyles $[1,2]$. Worldwide prevalence is expected to continue rising in both industrialized and developing countries $[2,3]$, with numbers projected to reach 552 million adults by 2030 $[1,4]$. DM2 is now a leading cause of death and disability and significantly increases risk for both macrovascular complications, such as atherosclerosis, and microvascular complications, such as retinitis, diabetic neuropathy, and renal disease [5]. DM2 is also strongly associated with elevated risk for other serious chronic conditions, including depression and dementia $[3,6]$. Cardiovascular disease (CVD) is the primary cause of morbidity and mortality in those with DM2
[7], accounting for at least $65 \%$ of deaths in this population [8]. In addition, the social and economic burden of DM2 is substantial and growing rapidly. For example, DM2 accounts for at least 10 percent of all healthcare expenses in the United States, making DM2 the single most costly chronic disease [9].

DM2 is typified by hyperglycemia in the presence of insulin resistance [6]. Other key related hemodynamic and metabolic abnormalities characterizing DM2 include elevated blood pressure, dyslipidemia, and chronic inflammation, as well as hypercoagulation and increased oxidative stress [5, 10-12]. Risk for DM2 rises with increasing age and is further elevated in certain racial and ethnic groups, including non-Hispanic blacks, Asians, Native Americans, and Pacific Islanders [13, 14]. However, while race, age, genetic predisposition, and other nonmodifiable factors are important in the pathogenesis of DM2, lifestyle factors, particularly, 
physical inactivity, overnutrition, and related obesity, are thought to be primarily responsible for the current global diabetes epidemic $[1,15]$. Other contributing lifestyle-related factors include chronic stress, impaired sleep, and smoking $[2,10,16,17]$. In fact, lifestyle factors may account for $90 \%$ of incident diabetes cases [2] and are significant predictors of DM2-related complications and mortality [18]. Thus, a central element of DM2 care is lifestyle management, which is considered critical to the prevention of acute complications and the reduction of risk for long term complications [6].

A central goal in DM2 management is the reduction of blood glucose levels, which has been shown to reduce risk of microvascular and possibly certain other complications $[3,5,6]$. However, while improving glycemic control remains a priority, the critical importance of multifactorial DM2 management has been increasingly emphasized in recent years, reflecting the complex constellation of factors that underlie the development of DM2 and its sequelae $[3,6]$. In particular, reducing CVD risk factors is crucial to effective DM management [6]. Additional goals include reducing DM-related distress, alleviating depression, and enhancing emotional well-being and quality of life, factors that are important predictors of glycemic control, complication rates, treatment adherence, and other outcomes [6, 19-24].

In light of the above, identifying sustainable lifestyle interventions with the potential to improve multiple factors of relevance to the management of this complex illness is of clear importance. Mind-body practices such as yoga, which capitalize on the ability of the mind to enhance physical health (and vice versa), appear particularly suited for addressing multifactorial conditions. Yoga is a traditional mind-body system originating in India over 4000 years ago [25]. In recent decades, the practice of yoga has been rising in both developed and developing countries worldwide [2629], and the field of yoga therapy is now growing rapidly [30]. The goals of yoga, a Sanskrit term meaning "yoke or union," do not center primarily on physical fitness, but rather on integration of mind, body, and spirit, cultivation of balance, calm, harmony, and awareness, and, in classic yoga traditions, the attainment of selflessness and spiritual enlightenment [25, 26]. Of the several major branches of yoga, the most widely practiced forms include Raja (royal or classical) yoga and the closely related Hatha yoga, sometimes known as the yoga of activity [25, 26]. Mantra yoga, emphasizing the use of specific sounds or chants to achieve mental and spiritual transformation, was popularized in the West by Maharishi Mahesh Yogi, the founder of Transcendental Meditation (TM). Hatha and Raja yoga emphasize specific postures (asanas), including both active and relaxation or restorative poses, as well as breath control (pranayama), concentration (dharana), and meditation (dhyana), with some schools focusing primarily on restorative/meditative practices. Hatha yoga, the branch of yoga most widely practiced in the US and other Western countries, also incorporates cleansing exercises, mantras or chants, and specific hand gestures (mudras). Originally developed to prepare the body for meditation, Hatha yoga itself encompasses many different styles, including Iyengar, Ashtanga, Integral, Kundalini, Viniyoga, Vinyasa, Kripalu, and Bikram yoga $[25,26]$.
A growing body of evidence suggests yoga practice may reduce risk for CVD and lead to improvements in physical health and well-being in a range of populations [31-37], potentially including those with DM2. In this systematic review, we critically evaluate available evidence from controlled trials regarding the effects of yoga-based programs on health-related outcomes in adults with DM2. We also outline major limitations in the current literature, briefly discuss possible mechanisms that may underlie observed benefits, and suggest directions for future research.

\section{Methods}

We searched eight databases via EbscoHost (Academic Search Complete, Alt HealthWatch, CINAHL with Full Text, Health Source: Nursing/Academic Edition, MEDLINE, PsycARTICLES, PsycINFO, and SPORTDiscus with Full Text) from their inceptions through March 2015 for original, scholarly articles reporting on controlled trials of yoga in the management of diabetes. The basic search strategy, (yoga OR yogic) AND diabetes, yielded 468 results; the same search conducted in the Cochrane Central Register of Controlled Trials resulted in 37 trials. A secondary search of the Physiotherapy Evidence Database (PEDro) for the terms yog ${ }^{*}$ AND diabetes yielded 34 articles. Titles and abstracts of the citations were scanned to identify potential articles for inclusion in this review. Potentially eligible papers were retrieved for more detailed review. In addition, we manually searched our own files, the citation sections of all identified articles, and the reference sections of recent review articles regarding diabetes and yoga.

Original, controlled studies were included if they evaluated the effects of yoga and yoga-based interventions on measures of glycemic control and insulin resistance, lipid profiles, body weight or composition, blood pressure, or other relevant outcomes, such as oxidative stress, nervous system function, cardiopulmonary function, mood and sleep impairment, or medication use. We excluded uncontrolled trials, cross-sectional studies, case series, and case studies, as well as trials that were published only in dissertation or abstract form. We also excluded articles that did not specifically target adults with diabetes, involve an intervention focused on yoga as the major component, study a yoga program of at least two week's duration, or report quantitative outcome data. Studies targeting populations with type 1 diabetes were also excluded, as the etiology and management for type 1 diabetes differ from those for DM2.

We categorized clinical measures and outcomes evaluated into several domains. For each domain, we summarized findings from relevant studies. Study quality was evaluated using the PEDro scale, which scores papers based on the following 10 criteria: (1) subjects were randomly allocated to groups, (2) allocation was concealed, (3) the groups were similar at baseline regarding the most important prognostic indicators, (4) there was blinding of all subjects, (5) there was blinding of all therapists who administered the therapy, (6) there was blinding of all assessors who measured at least one key outcome, (7) measures of at least one key outcome were obtained from more than $85 \%$ of the subjects 
initially allocated to groups, (8) all subjects for whom outcome measures were available received the treatment or control condition as allocated or, where this was not the case, data for at least one key outcome was analyzed by "intention to treat," (9) the results of between group statistical comparisons are reported for at least one key outcome, and (10) the study provides both point measures and measures of variability for at least one key outcome. One point is awarded for each criterion reported in the paper. In addition, PEDro assesses whether study eligibility criteria were specified; however, as this does not address statistical or internal validity, no points are awarded for this criterion. Whenever available, we reported the score given in the PEDro database; when the study was not in PEDro, we assigned a score based on the PEDro instructional materials (http://www.pedro.org.au/wp-content/uploads/PEDro_scale .pdf). In our discussion of findings, we also considered recent meta-analyses of RCTs targeting populations with diabetes.

Each eligible study identified was classified into one of two design categories: randomized controlled trials (RCTs) or nonrandomized controlled trials (NRCTs). In RCTs, each participant is randomly assigned to one of at least two groups: an active intervention (i.e., yoga) group and one or more comparator groups. In NRCTs, the experimental design and analytic strategy are similar to that of RCT's, but the allocation to a given treatment is not performed randomly. Articles were selected and data extracted and evaluated by both authors; conflicts were resolved by discussion and consensus.

\section{Results}

Of 539 potentially relevant abstracts and citation indices scanned, 159 potentially eligible papers were identified for closer review. A total of 33 papers reporting findings from 25 original studies, including 13 nonrandomized controlled trials (NRCTs) [38-50] and 12 randomized controlled trials (RCTs) [51-70], representing a total of 2170 enrolled participants met our eligibility criteria and were included in this review.

Of the 25 studies, 24 (96\%) specified adults with DM2; in the one remaining investigation, participants were recruited from a diabetes clinic, but authors did not specify type of diabetes (Table 1). Age range was wide in most studies, with some specifying no upper age limit $[57,60-62,65]$. Of the 23 studies that specified age cut-offs (12 NRCT, 11 RCTs), 8 (34.8\%) excluded patients under 30/35 years of age; an additional 10 (43.5\%) excluded those under $40 / 45$ years of age. While several studies included adults over the age of 65 [61, $62,66-70]$, only one study specifically targeted elder adults $[61,62]$. All but two studies $[51,52,60]$ included participants of both genders; likewise, only two studies clearly specified exclusion of patients on anti-DM medications [38, 43]. Patients ranged from newly or recently diagnosed DM2 to those diagnosed with DM2 for 10 years or more, often within the same study, with stated exclusion and inclusion criteria varying from study to study. Of the 17 studies with sufficient detail to allow determination, all but $2[45,65]$ excluded patients with significant complications (Table 1), although, again, exclusion conditions were not uniform across studies.

All but 2 were published in 2000 or later, with the majority (56\%) being published in the last 5 years. Most $(80 \%)$ of the studies were conducted in India. The vast majority, $76 \%$, were moderate-sized studies that included over 40 participants, with $44 \%$ including 61 or more participants and 8 (32\%) including at least 100 participants. Yoga interventions ranged from 15 days [60] to 12 months [69] in duration, with $72 \%$ including at least 12 weeks of practice (Table 1). Programs varied substantially in practice frequency, intensity, and content, including, for example, a 3-month Hatha yoga program in which participants attended 1-2 classes/week [65], a 90-day program of daily deep yoga relaxation practice (yoga nidra) [64], a 6-month Sudarshan Kriya (SKY) rhythmic breathing program, with classes once/week and daily home practice [56], and 3- to 12-month comprehensive yoga program with practice 6 to 7 days/week [57]. Ninety-two percent of the yoga programs incorporated active asanas or yoga poses. Comparator conditions also varied widely, from wait list/no treatment $[43,65]$, standard care [39-42, 45-51, 54-56, 58, 63-66, 70], and/or group education [61] to a comprehensive conventional exercise program $[57,60,66,69]$. Three studies included more than one comparator group $[38,63,66]$. For some studies, standard care included only medications [39, $40,48,49,58,63,64]$, whereas in others, standard care also included a special diet $[38,41,42,47,50,54,56,70]$ and/or walking or other exercises $[47,48,50,54-56]$.

3.1. Effects of Yoga on Metabolic, Anthropometric, and Hemodynamic Indices. Twenty-five eligible studies assessed the potential impact of yoga-based programs on one or more anthropometric or physiologic markers of importance to diabetes management and prognosis, including measures of glucose tolerance and insulin resistance, lipid profiles, body weight and composition, and blood pressure. Findings of these studies are summarized in Table 2 and are discussed briefly below.

3.1.1. Measures of Glycemia and Insulin Resistance. Twentyfour studies investigated the effects of yoga on markers of glycemia and insulin resistance, with all but two documenting significant, postintervention improvement in one or more measures following the practice of yoga either alone or in combination with other therapies. All but two programs [55, 64] incorporated active yoga asanas or poses; interventions ranged in duration from 15 days [60] to 12 months [69]. Of the 12 NRCTs (total $N=1090$ participants), all reported significant improvement in one or more indices of glycemia/insulin resistance; reductions in postprandial blood glucose (PPBG) [40, 42-46, 48], fasting blood glucose (FBG) [38-49], fasting insulin [48], fructosamine [38], and glycosylated hemoglobin (HbAlc) $[39,41-45,49]$ were observed among participants completing a yoga-based intervention compared to controls receiving no treatment [43], standard care [39-42, 44-46, $48,49]$, standard care with light exercise [47], or a low fat vegetarian diet alone [38].

Of the 12 RCTs evaluating the effects of yogic practices on indices of glycemia and insulin resistance $(N=1040$ 
TABLE 1: Characteristics of eligible studies investigating the effects of yoga-based programs in adults with diabetes (25 controlled trials, including 12 randomized (RCTs) and 13 nonrandomized (NRCTs)).

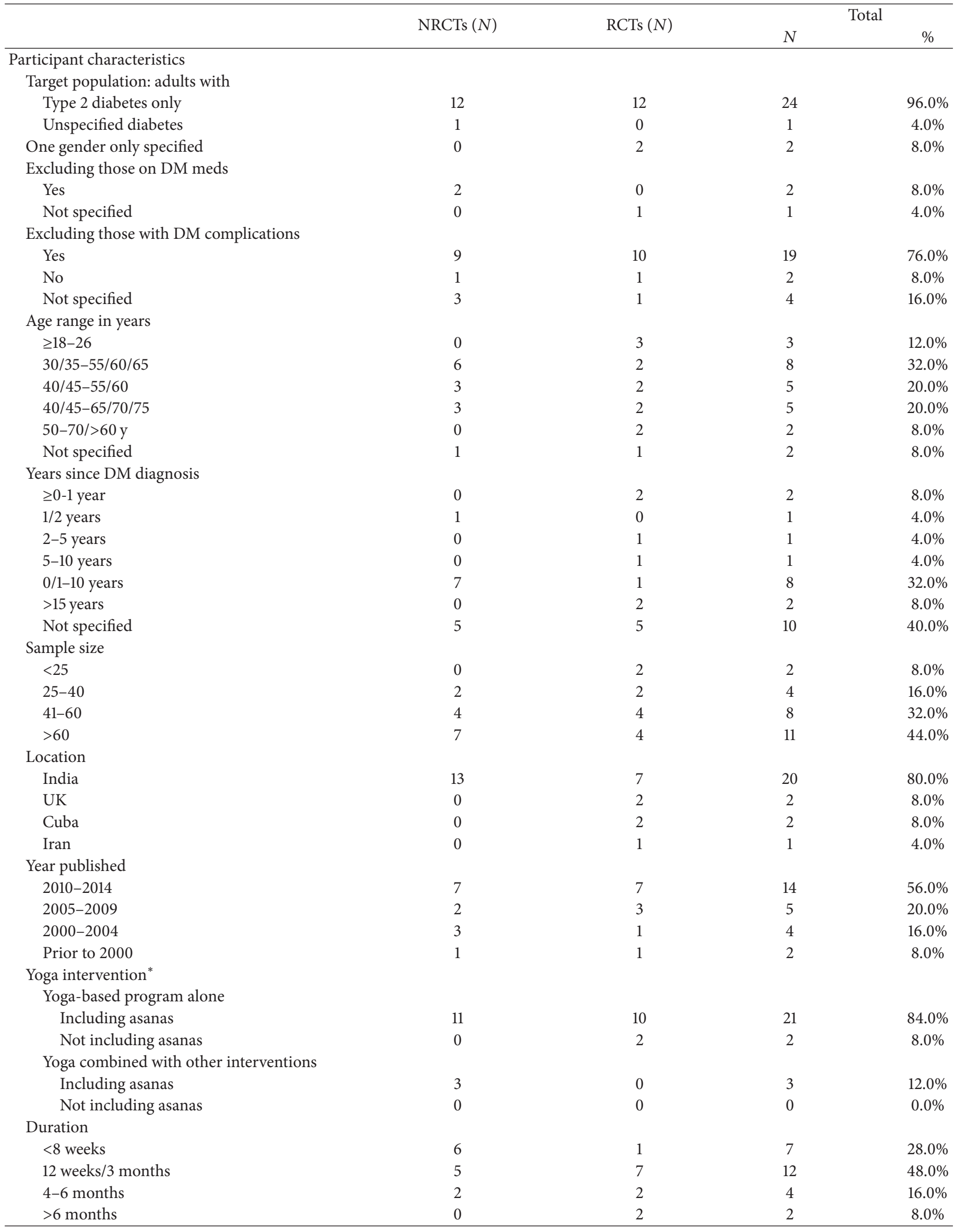


TABle 1: Continued.

\begin{tabular}{|c|c|c|c|c|}
\hline & \multirow{2}{*}{ NRCTs $(N)$} & \multirow{2}{*}{$\operatorname{RCTs}(N)$} & \multicolumn{2}{|c|}{ Total } \\
\hline & & & $N$ & $\%$ \\
\hline \multicolumn{5}{|l|}{ Frequency of practice ${ }^{\ddagger}$} \\
\hline$<3 \mathrm{x} /$ week & 0 & 1 & 1 & $4.3 \%$ \\
\hline $3 \mathrm{x} /$ week & 1 & 3 & 4 & $17.4 \%$ \\
\hline $4-5 \mathrm{x} /$ week & 2 & 1 & 3 & $13.0 \%$ \\
\hline $6-7 \mathrm{x} /$ week & 8 & 7 & 15 & $65.2 \%$ \\
\hline \multicolumn{5}{|l|}{ Program structure ${ }^{¥ ¥}$} \\
\hline Classes only & 9 & 7 & 16 & $69.6 \%$ \\
\hline Classes combined with home practice & 1 & 5 & 6 & $26.1 \%$ \\
\hline Training session combined with home practice & 1 & 0 & 1 & $4.3 \%$ \\
\hline \multicolumn{5}{|l|}{ Comparison condition ${ }^{* *}$} \\
\hline Usual care/no treatment & 11 & 8 & 19 & $76.0 \%$ \\
\hline Attention control & 0 & 1 & 1 & $4.0 \%$ \\
\hline Active comparator & 3 & 5 & 8 & $32.0 \%$ \\
\hline$>1$ control & 1 & 2 & 3 & $12.0 \%$ \\
\hline
\end{tabular}

*Including two yoga-based interventions tested within the same study [38].

${ }^{\ddagger}$ Practice frequency not specified in 2 NRCTs $[39,40]$.

¥¥ Information on program structure lacking in two NRCT’s [39, 41].

${ }^{* *}$ Numbers add up to more than 25 , as 3 studies included more than one comparator.

participants), 10 reported significant reductions in at least one measure (Tables 2 and 3). Reported improvements again included significant declines in PPBG [53, 55, 63, 64], FBG $[51,59-61,63,64,66,70]$, insulin [51], and HbAlc $[61,66,70]$ in those assigned to a yoga-based program versus standard care $[51,55,58,63,64,66,67,70]$, group education [61], or brisk walking [60]. Two additional trials observed significant beneficial changes in PPBG, FBG, and HbAlc following a comprehensive yoga program $[57,66]$ that were similar to those detected in participants assigned to a relatively intensive exercise intervention $[57,66]$.

In contrast, two RCTs documented no significant changes in these parameters. In a small 12-month Cuban trial of 40 adults with uncomplicated DM2, yoga group participants showed modest declines in FBG (7\%) that did not differ significantly from those of the exercise group (1.7\%) [69]. Similarly, a British study of 59 participants with DM2 showed no significant improvements either in glucose control [65], findings that may be in part attributable to low rates of participant compliance $(50 \%$ class attendance and $0 \%$ performance of home practice).

Collectively, $92 \%$ of the controlled trials reviewed, including 12/12 NRCTs and 10/12 RCTs, reported improvements in glucose control with yoga-based programs that were statistically and clinically significant, suggesting that yogic practices may improve glucose control in adults with DM2. However, between group comparisons were not reported in ten studies (7 NRCTs [38, 40,43, 44, 46-48], 3 RCTs $[60,63,64]$ ), and, as discussed below, additional methodologic or other limitations plagued most trials, hindering interpretation of findings. Magnitude of effects varied substantially among studies, from relatively modest to very large improvements depending on the study design, population, and comparator group (Table 3 ).
3.1.2. Lipid Profiles. Of the 25 studies included in this review, 16 ( $N=1575$ total participants) examined the effects of yoga programs on lipid profiles in those with DM2, with all but one trial [65] reporting significant improvement in one or more lipid indices (Tables 2-4). Studies all incorporated active yoga asanas and varied in length from 40 days [42] to 12 months [69]; in all except one trial [65], participants assigned to the yoga intervention practiced at least 3 days/week. As indicated in Table 2, all 8 NRCTs ( $N=737$ participants) reported significant improvements in lipid profiles, including reductions in levels of total cholesterol (TC), low-density lipoprotein cholesterol (LDL), very low-density lipoprotein cholesterol (VLDL), and triglycerides (Tg), and increases in high-density lipoprotein cholesterol (HDL) relative to standard care [38-40,42, 44, 48, 49], or standard care with light exercise [47].

Likewise, 7 of the 8 RCTs (totaling 838 participants) reported significant beneficial changes in serum lipids following completion of a yoga-based program. These included significant declines in TC $[58,61,63,66,69]$, LDL $[57,58$, $61,63,69]$, and $\operatorname{Tg}[51,58,61,69]$ and significant increases in HDL $[57,58,61,63,66,69]$ relative to standard care $[51,58$, $63,66]$, group education [61], or a moderate intensity exercise program $[57,69]$. Only one RCT, a 12-week UK study of 59 adults, reported no significant improvement in any of the serum lipids following the yoga program [65], findings, again, likely in part attributable to poor participant compliance.

Overall, 94\% of the studies reviewed reported improvements in lipid levels following completion of yogic programs of varying intensity and duration. Again, reported changes were clinically as well as statistically significant, with magnitude of effects varying substantially with the study and target population (Table 3 ). However, again, interpretation of findings is hampered by the lack of between group comparisons in 


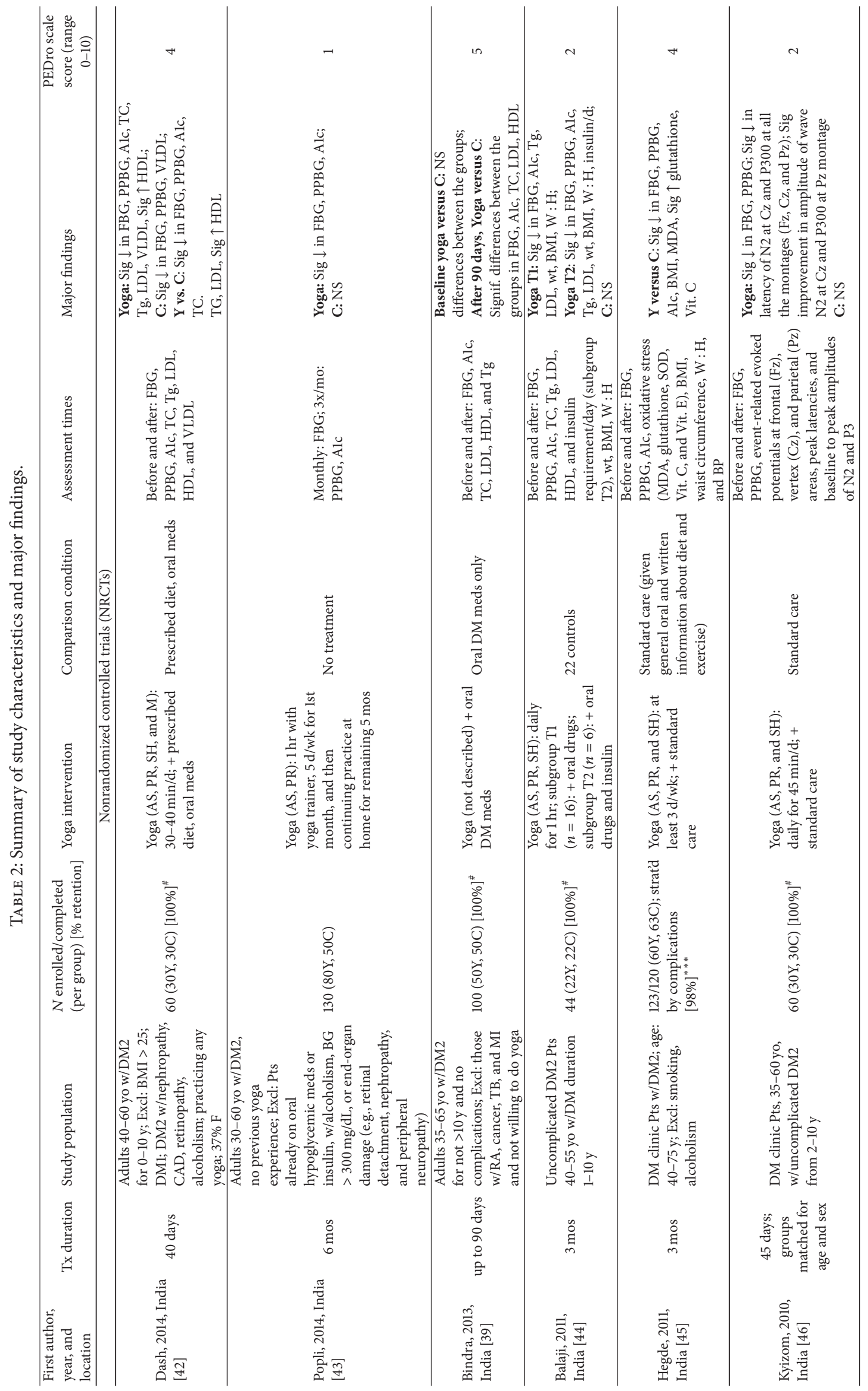




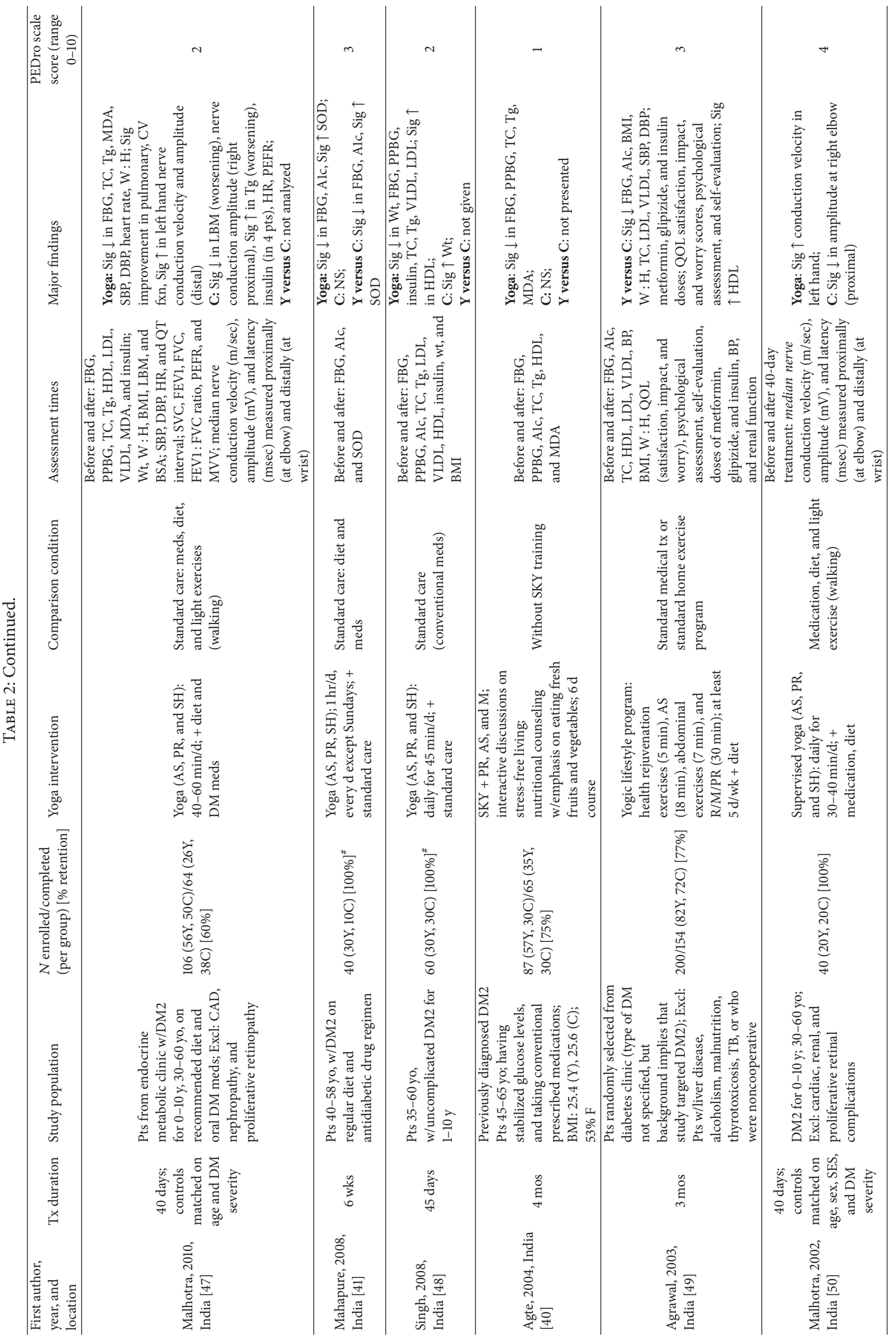




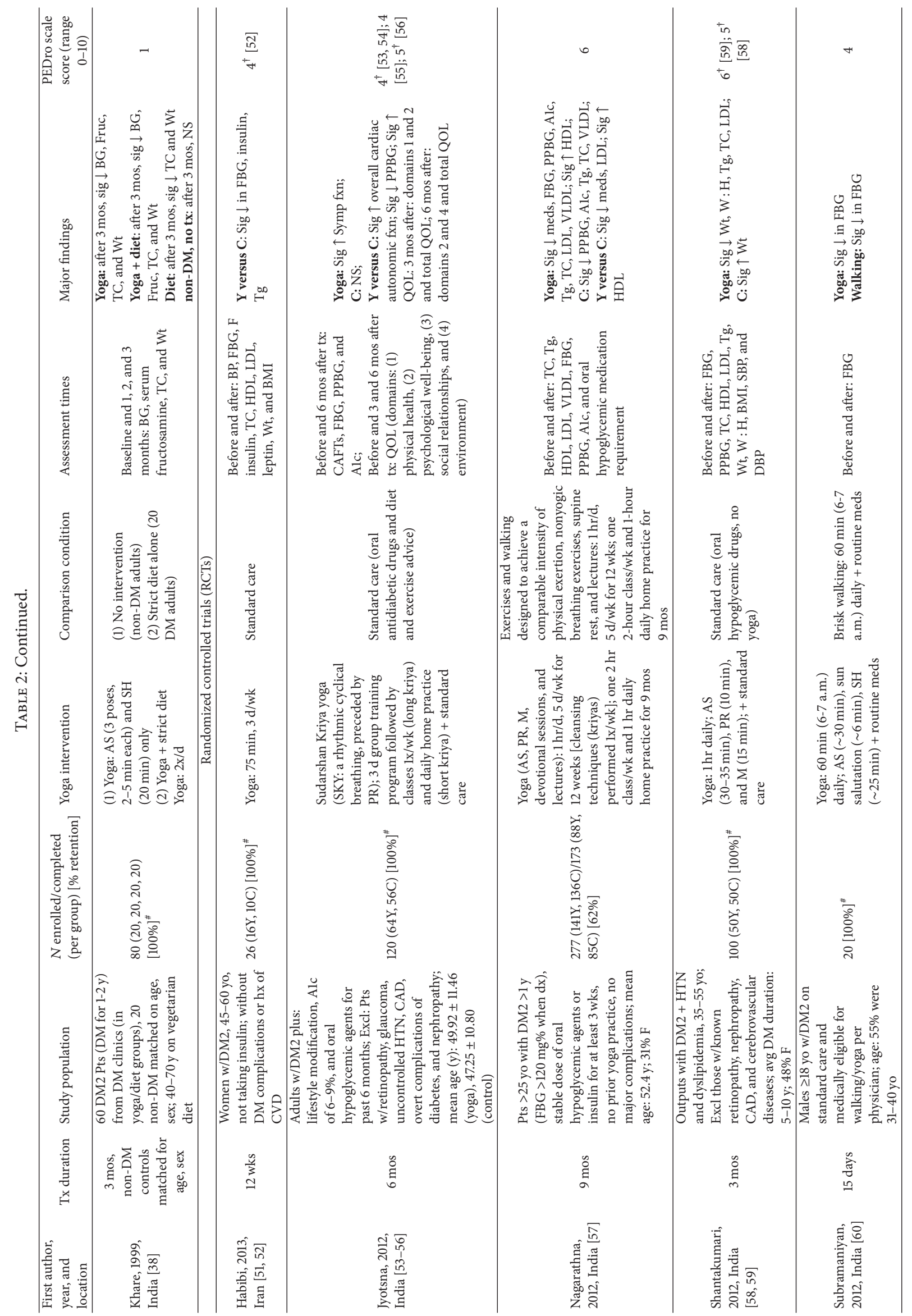




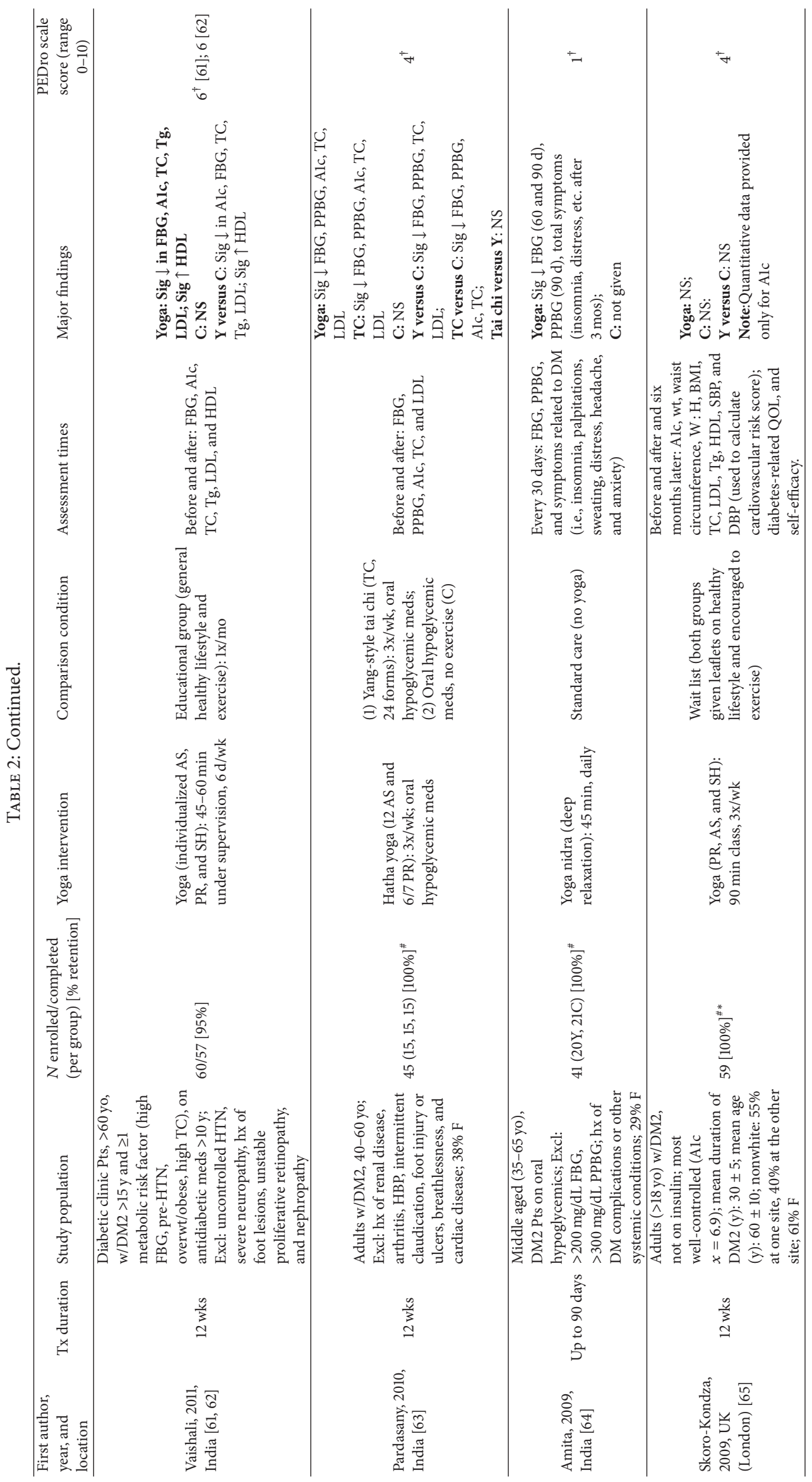




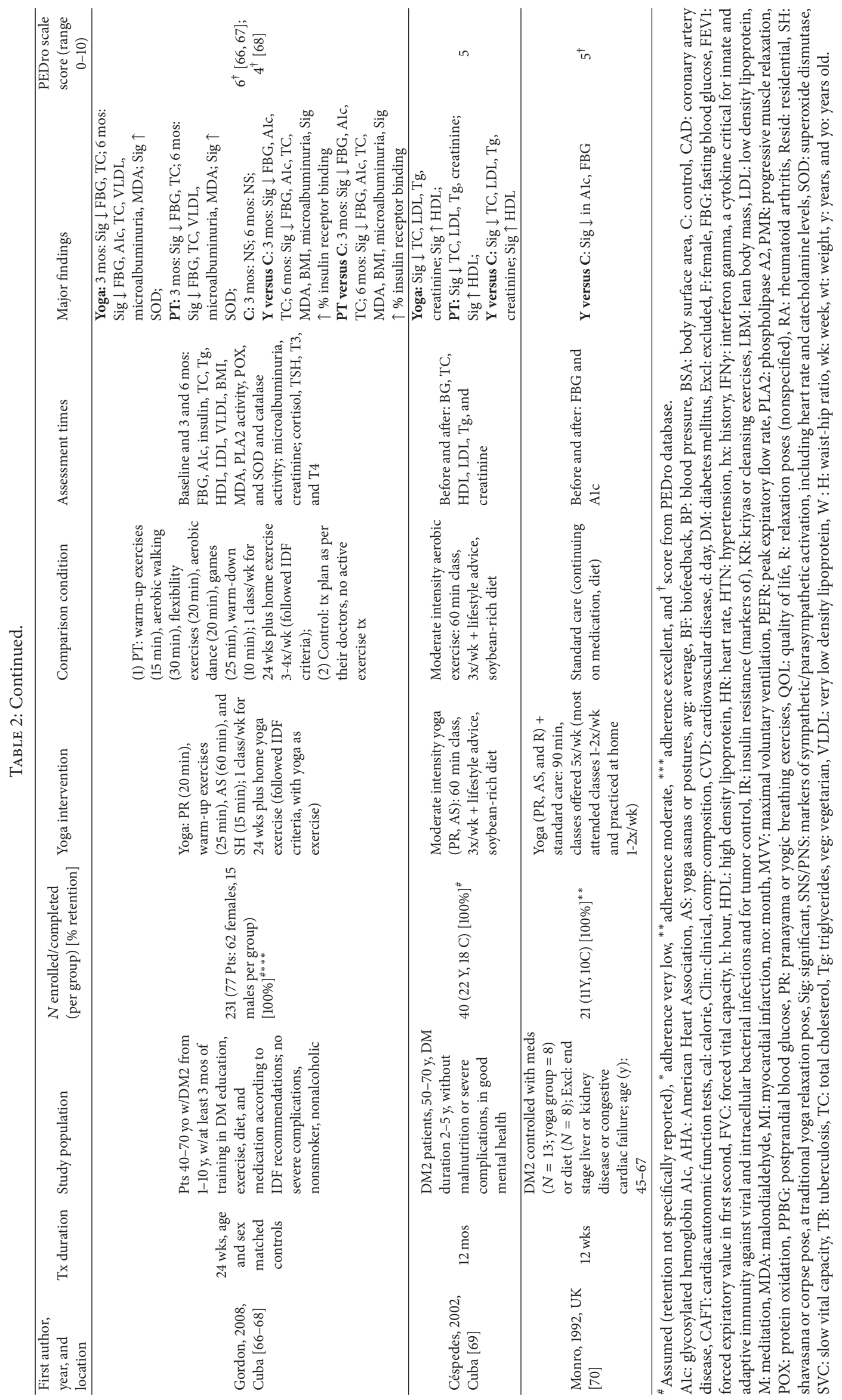




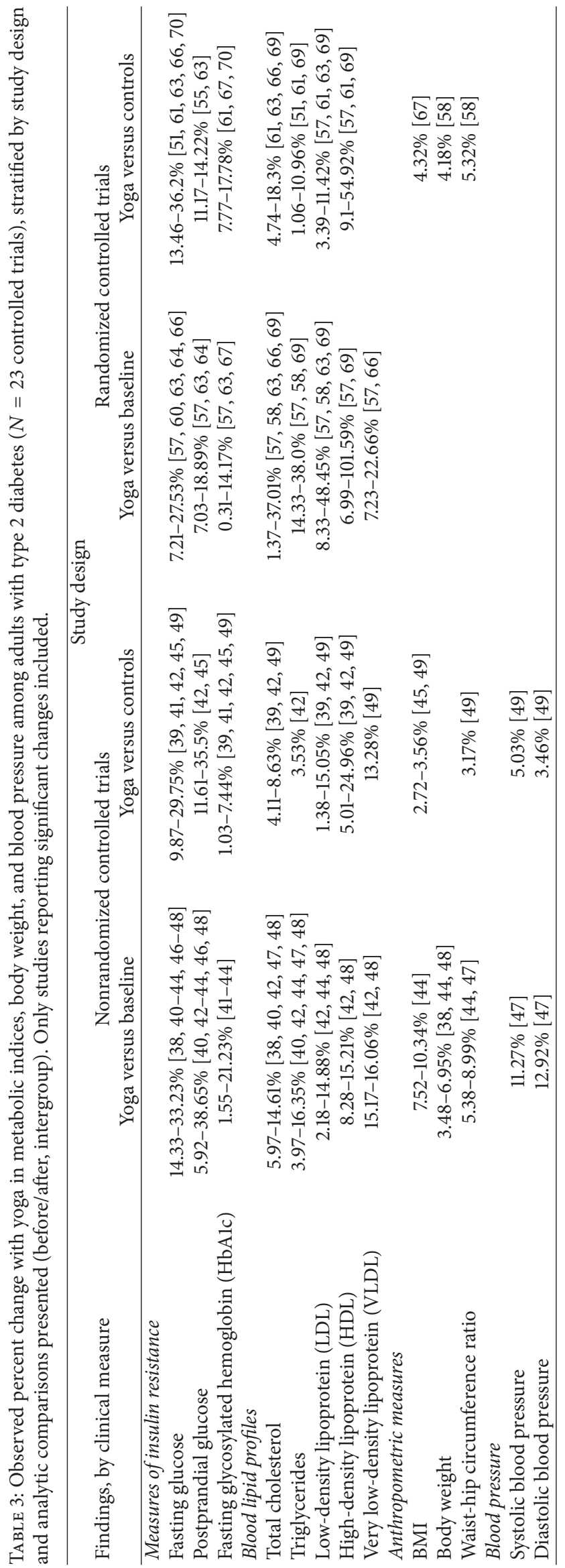




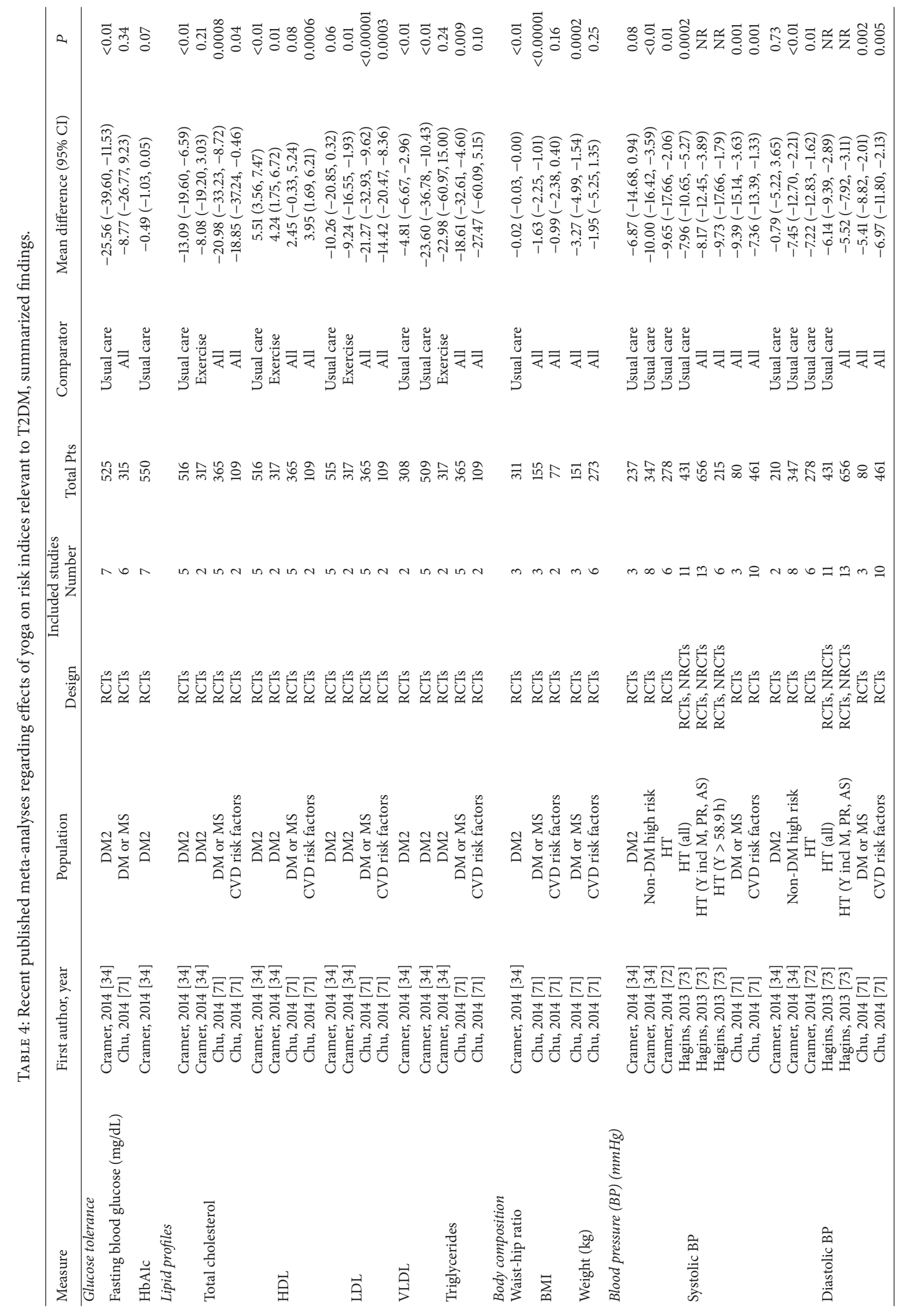




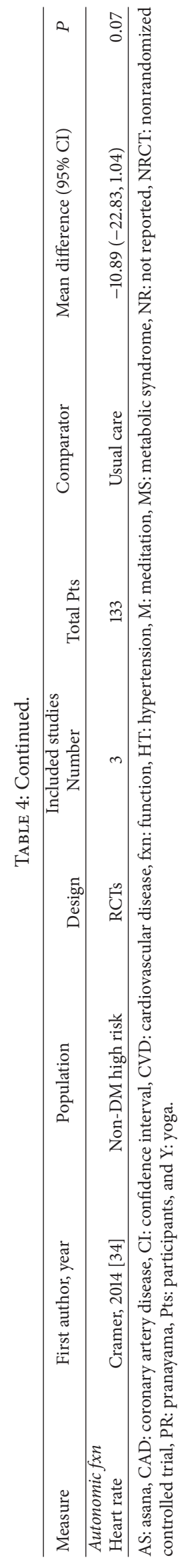


several studies, as well as other design, methodologic, and/or reporting limitations characterizing many of the studies, discussed in more detail below.

\subsubsection{Body Weight and Composition. Nine controlled trials} have evaluated the potential influence of yoga on body weight and composition in adults with DM2, including 6 NRCTs and 3 RCTs; again, all but one study [65] (89\%) documented improvements in those assigned to a yoga intervention. All of the yoga programs included active asanas. Trials ranged from 40 days [47] to 6 months [66] in duration, and, in all but the one trial reporting no significant benefits [65], participants practiced at least 3 times per week. Of the 6 NRCTs $(N=613$ total participants), all reported significant beneficial changes in at least one measure of body composition, including reductions in weight $[38,44,48]$, body mass index (BMI) [44, $45,48,49]$, and waist-hip ratio $[44,47,49]$ relative to standard care $[38,44,45,48,49]$ or standard care and light exercise [47]. Likewise, 2 of the 3 RCTs ( $N=390$ total participants), including a 6-month Cuban study in 231 adults [66] and a 3-month Indian trial of 100 adults [58], reported significant decreases in weight [58], BMI [66], and waist-hip ratio [58] in participants assigned to a yoga program versus standard care; yoga group improvements were again comparable to those documented following a moderate intensity physical activity program [66]. In contrast, a UK study of 59 adults showed no significant reduction in weight or BMI relative to standard care [65], although poor participant adherence renders interpretation of findings problematic.

3.1.4. Blood Pressure. Only 5 of the 25 controlled trials targeting adults with DM2 evaluated the potential effects of yogic practices on blood pressure, including 3 NRCTs and 2 RCTs ( $N=$ total of 588 participants). Trials varied in length from 40 days to 3 months, and all included active asanas. Three of the five studies, two NRCTs and one RCT [59], in Indian adults showed significant drops in systolic and diastolic blood pressure relative to standard care $[49,59]$ or standard care combined with walking [47]. Another Indian study of 123 adults [45] showed similar, but not statistically significant, declines in blood pressure in participants assigned to the yoga versus the standard care group. One exploratory RCT of British adults [65] did not present any quantitative data on blood pressure but reported no significant changes following a 12-week versus standard care program.

3.2. Observed Effects of Yoga on Other Pertinent Indices. There is mounting evidence, reviewed briefly below, that yoga may improve other risk indices pertinent to the DM2 management as well, including oxidative stress, impairments in mood and sleep, nervous system and pulmonary function, and medication usage. Oxidative stress, an imbalance between free radicals and antioxidants, has been strongly implicated in the development of DM2 and diabetes-related complications [74, 75]. For example, the oxidative imbalance that often characterizes DM2 can lead to prothrombotic changes, endothelial dysfunction, and chronic vascular inflammation, exacerbate insulin resistance and associated hyperglycemia, and ultimately promote organ damage; these reciprocally related changes are thought to mediate many of the atherosclerotic and thrombotic alterations associated with the metabolic syndrome and to play a central role in the pathogenesis and progression of diabetes and CVD [10,75-78]. Thus, interventions that reduce oxidative stress in those with diabetes may aid in improving risk profiles and decreasing diabetes-related complications. To date, at least five controlled trials have examined the effects of yoga on measures of oxidative stress in adults with DM2. Yoga interventions all incorporated active asanas and ranged from 40 days to 6 months in duration; all indicated beneficial changes with yoga (Table 2). For example, four NRCTs in Indian adults documented improvements in indices of oxidative balance; these included significant declines in MDA $[40,45,47]$ among participants completing a 40-day [47] to 4-month yoga program [40] versus those assigned to standard care $[40,45]$ or standard care with light exercise [47]; in their 3-month study of 123 adults, Hegde et al. also reported significantly greater increases in serum levels of the antioxidants glutathione and Vitamin $\mathrm{C}$ in the yoga versus standard care group [45]; likewise, Mahapure et al. noted significantly greater increases in superoxide dismutase (SOD) levels in the yoga group relative to the standard care control in their 6-week study [41]. Additionally, in an RCT of 231 Cuban adults, those assigned to a 24 -week yoga program showed increases in activity of the antioxidant, SOD, and reductions in malondialdehyde (MDA), an estimate of lipid oxidative damage, that were comparable to those completing a 24-week conventional exercise program, and greater than participants assigned to standard care [66].

Mood and sleep impairment, common comorbid conditions in those with DM2, are reciprocally related to the development and progression of DM2 $[16,79]$. For example, depression and sleep disturbance, both characterized by dysregulation of the hypothalamic-pituitary-adrenal (HPA) axis, sympathetic overactivity, and reduced cardiovagal tone [80-88], have been shown to increase subsequent risk for prediabetes [89] and incident diabetes [16, 90-98], impair metabolic control in persons with diabetes [99-104], and lead to excess morbidity and mortality $[10,22,76,93-95$, 105-109]. However, although there is substantial evidence to suggest that yogic practices are helpful in reducing symptoms of depression and anxiety [35, 37, 80, 110, 111], alleviating stress $[10,80,112,113]$, improving sleep $[80,110,111,114-$ $119]$, and enhancing psychological well-being $[80,112]$ in both healthy and clinical populations, few controlled trials have examined the effects of yoga on these endpoints in populations with DM2. Our search identified only four that specifically assessed indices of psychological status, including one NRCT [49] and three RCTs [53, 55, 64, 65]; significant improvements were noted with yogic practices in all but one study (Table 2). These included beneficial changes in multiple domains of quality of life $[49,53,55]$, measures of psychological well-being [49], and symptoms of distress [64]. Studies regarding the effects of yoga on sleep in those with DM2 are even fewer. We identified only one trial of DM2 patients, an RCT conducted in India, that examined the effects of yoga on any measure of impaired sleep; in this 
study of 41 adults, participants completing a program of daily yoga nidra (a deep relaxation yoga practice) experienced a reduction in the prevalence of insomnia, from 43 to 5\% [64].

3.2.1. Nervous System Function. Autonomic nervous system dysfunction, a correlate of obesity and poor cardiorespiratory fitness, has been strongly and bidirectionally related to insulin resistance and hypertension, implicated in the development of diabetes [120,121] and CVD [122], and associated with increased risk for morbidity and mortality in individuals with DM2 $[123,124]$. While more than 30 controlled studies, including 17 RCTs, have evaluated the effect of yoga on markers of sympathetic/parasympathetic activation and cardiovagal function $[125,126]$, our search identified only three that targeted adults with DM2, all conducted in India. These include two NRCTs $[47,127]$ assessing the effects of 3month program of simple pranayama exercises [127] and a 40-day comprehensive yoga intervention [47] and an RCT of SKY yoga (a cyclical breathing practice) in adults enrolled in a diabetes lifestyle modification program that included daily brisk walking [54, 55] (Table 2). Participants who completed a pranayama program showed significant improvements in multiple indices of cardiac autonomic function [47, 55] and significant reductions in heart [47] and respiratory rate [127], relative to controls receiving standard care $[55,127]$ or standard care combined with exercise [47]. These findings suggest that yogic practices may promote a reduction in sympathetic activation, enhancement of cardiovagal function, and a shift in autonomic nervous system balance from primarily sympathetic to parasympathetic in adults with DM2. In addition, findings of three recent NRCTs suggest that yoga may also help mitigate the central and peripheral nervous system damage associated with DM2. For example, following completion of a 45-day daily yoga program, participants showed significant declines in the latency of event-related potentials (ERP), a marker of higher brain, including cognitive and memory function, and increases in ERP amplitude compared to those assigned to standard care [46]. Likewise, adults with DM2 who completed a 40day yoga intervention showed significant improvements in certain measures of median nerve conduction velocity and amplitude relative to controls $[47,50]$, although incomplete information and lack of between group comparisons limit interpretation of findings.

3.2.2. Pulmonary Function. Compromised lung function is both an important complication $[128,129]$ and a significant predictor [130] of DM2 and has been inversely associated with insulin resistance [128] and glycemic exposure [129, 131]. While studies in adults with DM2 are limited, available data from two NRCTs support a possible beneficial influence of yogic practices on pulmonary function in this population [47, 127]. Reported improvements included significant increases in forced expiratory volume, forced vital capacity, peak expiratory flow rate, and maximum voluntary ventilation following completion of a 3-month program of simple yogic breathing exercises [127] or a 40-day comprehensive program [47].
3.2.3. Medication Use. Three controlled trials to date (two NRCTs [44, 49], one RCT [57]) have shown significant reductions in diabetes medication use in patients completing a three- $[44,49]$ to nine-month [57] yoga program relative to those assigned to standard care $[44,49]$ or a comprehensive exercise program [57]. Some of these declines were quite substantial. For example, in their trial of 154 adults with diabetes, Agrawal et al. reported 26 to $40 \%$ reductions in medication use in the yoga group at the 3-month follow-up [49].

\section{Discussion}

Overall, findings of these studies suggest that yoga-based practices may have significant beneficial effects on multiple factors important in DM2 management and prevention, including glycemic control, insulin resistance, lipid profiles, body composition, and blood pressure. These findings are further supported by recently published meta-analyses regarding the effects of yoga on specific CVD risk factors of relevance to DM2, detailed in Table 4 [34, 71-73]. For example, in subanalyses restricted to RCTs in adults with DM2, Cramer et al. reported greater average declines of $26 \mathrm{mg} / \mathrm{dL}$ in FBG and $0.5 \%$ in HbAlc, greater mean reductions of $13 \mathrm{mg} / \mathrm{dL}$ in TC, $10 \mathrm{mg} / \mathrm{dL}$ in LDL, $5 \mathrm{mg} / \mathrm{dL}$ in VLDL, and $24 \mathrm{mg} / \mathrm{dL}$ in Tg levels, and a higher average increase of $6 \mathrm{mg} / \mathrm{dL}$ in HDL in participants assigned to a yoga versus standard care group [34]. Notably, the authors also found significantly greater reductions in LDL $(9 \mathrm{mg} / \mathrm{dL})$ and higher increases in HDL levels $(4 \mathrm{mg} / \mathrm{dL})$ in the yoga versus conventional exercise group. Likewise, Cramer et al. also reported greater mean reductions in waist-hip ratio in participants with DM2 assigned to a yoga versus a standard care group [34]. Similarly, in a subanalysis of RCTs in adults with diabetes or metabolic syndrome, Chu et al. reported a mean BMI reduction of over $1.6 \mathrm{~kg} / \mathrm{m}^{2}$ in those in the yoga versus control group [71]. While studies regarding the effects of yoga on blood pressure in adults with DM2 remain few, recent metaanalyses regarding the effects of yoga on other populations at risk for CVD suggest that yoga may be beneficial for regulating blood pressure in patients with diabetes as well. For example, in subanalyses of RCTs limited to 8 trials of adults without frank diabetes but at high risk of DM2 and CVD (e.g., those with obesity, impaired glucose tolerance, or metabolic syndrome), the authors noted significant mean declines of $10 \mathrm{mmHg}$ in systolic blood pressure and $7.5 \mathrm{mmHg}$ in diastolic blood pressure in participants receiving a yoga intervention versus standard care [34]. Meta-analyses of controlled trials in adults with hypertension have yielded similar findings $[72,73]$, indicating significant declines of $8-10 \mathrm{mmHg}$ in systolic blood pressure and $6-7 \mathrm{mmHg}$ in diastolic blood pressure in the yoga versus standard care groups (Table 4).

More limited data suggest that yoga may also lower oxidative stress, decrease sympathetic activation and improve nervous system function, enhance pulmonary performance, mood, sleep, and quality of life, and reduce medication use in those with DM2. Relatively few studies have examined the effects of yogic practices on psychological status and sleep, 


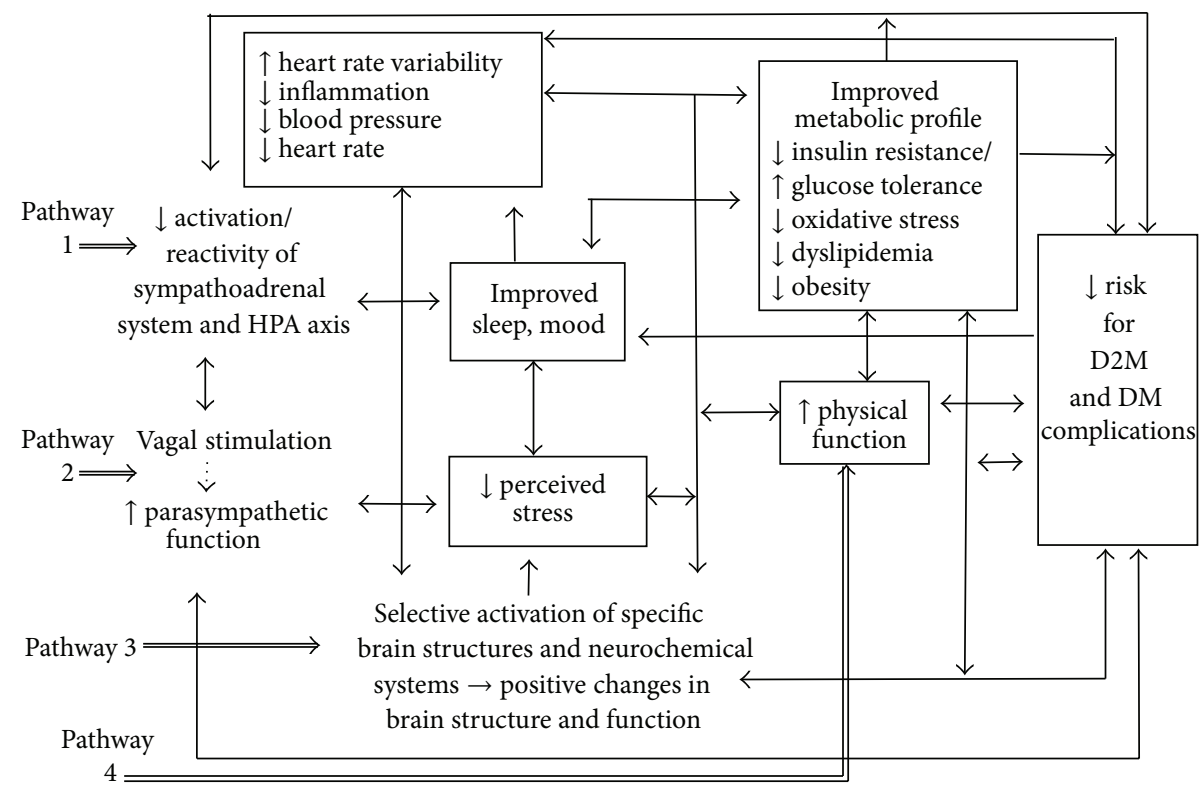

FIGURE 1: Some pathways by which yoga practices may influence outcomes in those with and at risk for type 2 diabetes (DM2). Figure adapted with permission from Hansen and Innes [132].

which are frequently compromised in DM2 and significant contributors to diabetes progression. Likewise, studies evaluating the effects of yoga on clinical outcomes and certain emerging risk factors in DM2, including proinflammatory markers, remain sparse, and studies to determine optimal yoga dosing and program structure for different DM2 populations are lacking. As indicated in Table 1, the vast majority of controlled studies published to date regarding the effects of yoga for DM2 have been conducted in India, underscoring the need for rigorous trials in other racial/ethnic groups and in other developed and developing countries. Finally, as discussed in more detail below and reflected in the study quality scores (Table 2), the methodological limitations characterizing existing studies highlight the need for additional high-quality RCTs that address these concerns.

\section{Yoga and DM2: Possible Underlying Mechanisms}

Although the mechanisms underlying the apparent beneficial effects of yoga therapy on diabetes risk profiles are not yet well understood, mechanistic pathways are likely complex and interacting. The observed changes may occur through at least four pathways, as depicted in Figure 1 [10, 76, 113, 117, 125, 132].

First, yoga may lessen the negative impact of stress and promote multiple positive downstream effects on metabolic function, neuroendocrine status, and related inflammatory responses and, ultimately, reduce risk for CVD and other vascular complications, by enhancing well-being and reducing reactivity and activation of the HPA axis and the sympathoadrenal system $[10,76,113,132]$. For instance, recent controlled trials suggest even short term yoga training programs can reduce perceived stress, improve mood, and lower catecholamine and cortisol levels, cardiovascular response to stress, blood pressure, and other indices of sympathetic activation in both healthy and clinical populations, including adults with diabetes $[10,35,80,113,132-134]$. Sympathetic activation, HPA dysregulation, and psychological distress have also been linked to the development and exacerbation of several risk factors for DM2 [10, 76, 80, 83, 88, 132, $133,135,136]$, including insulin resistance, impaired glucose tolerance, hypertension, dyslipidemia, central obesity, and proinflammatory and prothrombotic states. These conditions have been shown to have adverse effects on metabolic control and neuroendocrine function and have also been implicated in elevated risk for DM2, CVD, and other vascular disorders $[10,76,80,83,88,132,133,135]$.

Second, yogic practices may shift the autonomic nervous system balance from primarily sympathetic to parasympathetic, by directly enhancing parasympathetic output, possibly via vagal stimulation $[76,122,137]$, resulting in positive changes in cardiovagal function and associated neuroendocrine, hemodynamic, and inflammatory profiles, in sleep and affect, and in related downstream metabolic parameters (Figure 1, pathway 2) [10, 80, 113, 133]. For example, recent controlled studies in adults with DM2, CVD, hypertension, and other chronic conditions, as well as in healthy populations, have shown yogic exercises to reduce resting heart rate, enhance baroreflex sensitivity, and increase heart rate variability, both immediately and following short term (612 weeks) yoga programs [10, 113, 133, 138, 139]. Heart rate variability, resting heart rate, and baroreflex sensitivity are widely used markers of cardiovagal autonomic function, parasympathetic activation, and autonomic balance $[76,120$, $140,141]$ and are thought to in part reflect stimulation of the vagus nerve $[76,122,137]$. These factors have been strongly linked to increased risk for DM2, as well as CVD [10, 76, 80, 142]. 
Third, yoga may also promote favorable changes in autonomic balance, memory and mood, neurological structure and function, and related metabolic and inflammatory responses by selectively activating specific brain structures and neurochemical systems related to attention and positive affect (Figure 1, pathway 3), as suggested by recent neurophysiological and neuroimaging research findings [143-146]. And finally, yogic practices may improve both metabolic and psychological risk profiles, support increased physical activity, enhance neuroendocrine function, improve body composition, and promote weight loss by increasing strength, overall fitness, and physical function (Figure 1, pathway 4). Yoga may also reduce CVD risk in other ways. For instance, by reducing stress and leading to improved sleep and mood, yoga may indirectly improve CVD risk profiles by leading to healthier lifestyle choices and enhanced self-care [147]. Yoga may also increase resilience to stress, a factor that has been linked to improved outcomes in DM2 [148, 149], although study findings to date have been inconsistent [150-152].

In addition, yoga may benefit those with DM2 indirectly by encouraging improvements in health-related attitudes and lifestyle choices $[147,153,154]$ and by providing a source of social support, a factor linked to improved diabetes self-care and clinical outcomes $[155,156]$. As suggested by recent studies of patients with heart failure, chronic obstructive pulmonary disease, and DM1 [157-159], yogic breathing practices may also, by increasing arterial and tissue oxygenation, alleviate underlying hypoxia and thereby enhance autonomic cardiac and respiratory function and related endpoints in adults with diabetes [157-160]. Finally, several recent genomic investigations in dementia caregivers $[161,162]$ and healthy adults $[163,164]$ suggest that yogic meditative practices can slow cellular aging and induce beneficial epigenetic changes in pathways regulating inflammation, oxidative stress, energy metabolism, insulin secretion, mitochondrial function, and other related factors; these changes may, in turn, help buffer the deleterious effects of stress, improve glucose control, enhance mood, sleep, and autonomic function, reduce blood pressure, and promote improvements in other related risk factors of relevance to DM2 management $[76,165,166]$.

\section{Study Limitations and Directions for Future Research}

This systematic review was limited in that it did not include unpublished studies, dissertations, or abstracts. In addition, some controlled trials of yogic practices may have been missed if the article did not make reference to the word yoga or yogic, although every effort was made to locate all relevant studies meeting our inclusion criteria. Many of the studies reviewed here suffer from methodological problems, poor reporting, and other limitations that render interpretation of findings challenging and the formation of definitive conclusions difficult. PEDro scores ranged from a low of $1[38,40,43,64]$ to a high of $6[57,59,61,62,66,67]$ points, out of 10 (Table 2). Importantly, participants were not randomly assigned to treatment in more than half of the studies, increasing risk for selection bias, and concealment of treatment allocation was reported in only $3[57,61,65]$ of the 25 trials. Among the 12 RCTs, only $2[57,65]$ specified how randomization was performed, and only 7 [52-56, 58, $59,61-63,66-68,70]$ demonstrated baseline comparability between the groups regarding the most important prognostic indicators. Interestingly, 8 [39, 44-50] of the 13 NRCTs also exhibited groups that were similar at baseline.

Given the nature of the intervention, blinding of the therapists and participants was likely not feasible; understandably, none of the studies satisfied these two PEDro criteria. This deficiency in blinding potential places even greater importance on ensuring that outcome assessors are blinded to group assignment. Unfortunately, blinded outcome assessment was specifically addressed in only two trials $[57,66,67]$, raising the possibility of information bias. Retention was not always reported and varied widely (from $60 \%$ to $100 \%$ ), with less than half of the studies ( $n=11[39,42$, $45,50,56,58-62,66,67,69,70]$ ) reporting outcome data from $85 \%$ or more of those initially assigned to a group. Reasons for dropout were rarely specified. Only five studies included specific data on participant adherence $[45,56,65,67,70]$, and even in these studies, adherence was highly variable, ranging from excellent $[45,67]$ to very poor [65]; moreover, for one study [53], adherence data were reported for only a subset of participants [56].

In some articles, details regarding analytic methods were sparse, and/or analyses were inadequate. For example, while all studies in this review included a comparator group, between group comparisons were not presented in 10 of the 25 studies, including $2[60,64]$ RCTs. In those studies documenting attrition, only eight $[39,42,50,57,59,60$, 65, 69] indicated all participants completed the study as designed or that intention to treat analyses had been conducted. Additionally, although all but two $[64,65]$ studies provided point measures and measures of variability, other data were often incompletely or confusingly presented. Furthermore, in many papers, the study population, participant recruitment, yoga-based intervention, and/or comparator condition were poorly described, rendering replication of these studies challenging and limiting conclusions. Most of the trials were relatively small, with $56 \%$ of them including 60 or fewer participants and $24 \%$ including 40 or less (Table 1), although, overall, sample size has been increasing in the last decade, especially the last 5 years.

While the investigations published to date have yielded overall positive results, the considerable heterogeneity in study design, intervention, comparator condition, and duration characterizing the 25 trials render direct comparisons across studies challenging. Participant characteristics also varied substantially, both within and between studies. Nonetheless, while this heterogeneity precludes specific recommendations for particular yoga programs or subpopulations, the consistently positive findings reported in multiple and varied samples of adults with DM2 suggest that yoga may be helpful for a broad range of patient groups. Of the 25 eligible controlled studies identified in this review, only five were conducted in countries other than India, including two in the UK, two in Cuba, and one in Iran. 
Rigorous trials in Western and many other countries, including many developing nations, where DM2 is now reaching epidemic proportions, remain few. It thus remains unclear if the positive findings reported in existing studies can be generalized to other populations, cultures, and/or regions. As indicated earlier, the mechanisms underlying the observed benefits of yoga are still poorly understood. Finally, rigorous dosing, cost-effectiveness, and long-term follow-up studies are lacking, as are trials assessing the effects of yoga on clinical endpoints such as diabetes-related morbidity and mortality. All of these areas warrant future research.

\section{Conclusion}

In conclusion, the findings of controlled trials published to date suggest that yogic practices may promote significant improvements in several indices of major importance in the management of DM2, including glycemic control, lipid levels, and body composition. More limited data suggest that yoga may also lower oxidative stress and blood pressure, enhance pulmonary and nervous system function, improve mood, sleep, and quality of life, and reduce medication use in adults with DM2. However, given the methodological limitations and heterogeneity of existing studies, findings must be interpreted with caution. Additional high-quality investigations are required to confirm and further elucidate the potential therapeutic benefits of standardized yoga programs in populations with DM2 [10, 132].

\section{Disclaimer}

The contents are solely the responsibility of the authors and do not represent the official views of West Virginia University or the National Institutes of Health.

\section{Conflict of Interests}

The authors declare that there is no conflict of interests regarding the publication of this paper.

\section{Acknowledgments}

This work was performed at West Virginia University and was supported by the National Center for Complementary and Alternative Medicine [Grant no. 1-K01-AT004108 to KEI] and West Virginia University (Faculty Incentive Award).

\section{References}

[1] D. W. Lam and D. LeRoith, "The worldwide diabetes epidemic," Current Opinion in Endocrinology, Diabetes and Obesity, vol. 19, no. 2, pp. 93-96, 2012.

[2] L. Chen, D. J. Magliano, and P. Z. Zimmet, "The worldwide epidemiology of type 2 diabetes mellitus-present and future perspectives," Nature Reviews Endocrinology, vol. 8, no. 4, pp. 228-236, 2012.

[3] S. E. Inzucchi, R. M. Bergenstal, J. B. Buse et al., "Management of hyperglycemia in type 2 diabetes: a patient-centered approach: position statement of the American Diabetes Association
(ADA) and the European Association for the Study of Diabetes (EASD)," Diabetes care, vol. 35, no. 6, pp. 1364-1379, 2012.

[4] International Diabetes Federation, IDF Diabetes Atlas, International Diabetes Federation, Brussels, Belgium, 2011.

[5] M. J. Fowler, "Microvascular and macrovascular complications of diabetes," Clinical Diabetes, vol. 29, no. 3, pp. 116-122, 2011.

[6] American Diabetes Association, "Standards of medical care in diabetes-2014," Diabetes Care, vol. 37, supplement 1, pp. S14S80, 2014.

[7] J. J. Joseph and S. H. Golden, "Type 2 diabetes and cardiovascular disease: what next?" Current Opinion in Endocrinology, Diabetes and Obesity, vol. 21, no. 2, pp. 109-120, 2014.

[8] American Heart Association, Cardiovascular Disease \& Diabetes, 2012, http://www.heart.org/HEARTORG/Conditions/ Diabetes/WhyDiabetesMatters/Cardiovascular-Disease-Diabetes_UCM_313865_Article.jsp/.

[9] M. Petersen, "Economic costs of diabetes in the US in 2012," Diabetes Care, vol. 36, no. 4, pp. 1033-1046, 2012.

[10] K. E. Innes and H. K. Vincent, "The influence of yogabased programs on risk profiles in adults with type 2 diabetes mellitus: a systematic review," Evidence-based Complementary and Alternative Medicine, vol. 4, no. 4, pp. 469-486, 2007.

[11] K. E. Wellen and G. S. Hotamisligil, "Inflammation, stress, and diabetes," Journal of Clinical Investigation, vol. 115, no. 5, pp. 11111119, 2005.

[12] J. S. Rana, M. Nieuwdorp, J. W. Jukema, and J. J. P. Kastelein, "Cardiovascular metabolic syndrome - an interplay of, obesity, inflammation, diabetes and coronary heart disease," Diabetes, Obesity \& Metabolism, vol. 9, no. 3, pp. 218-232, 2007.

[13] K.-H. Yoon, J.-H. Lee, J.-W. Kim et al., "Epidemic obesity and type 2 diabetes in Asia," The Lancet, vol. 368, no. 9548, pp. 1681$1688,2006$.

[14] P. Zimmet, J. Shaw, S. Murray, and R. Sicree, "Diabetes: an epidemic in full flight: forecasting the future," Bulletin of the International Diabetes Federation, vol. 48, pp. 12-16, 2003.

[15] F. B. Hu, "Globalization of diabetes. The role of diet, lifestyle, and genes," Diabetes Care, vol. 34, no. 6, pp. 1249-1257, 2011.

[16] F. P. Cappuccio, L. D’Elia, P. Strazzullo, and M. A. Miller, "Quantity and quality of sleep and incidence of type 2 diabetes: a systematic review and meta-analysis," Diabetes Care, vol. 33, no. 2, pp. 414-420, 2010.

[17] M. T. U. Barone and L. Menna-Barreto, "Diabetes and sleep: a complex cause-and-effect relationship," Diabetes Research and Clinical Practice, vol. 91, no. 2, pp. 129-137, 2011.

[18] C.-C. Lin, C.-I. Li, C.-S. Liu et al., "Impact of lifestyle-related factors on all-cause and cause-specific mortality in patients with type 2 diabetes: the Taichung diabetes study," Diabetes Care, vol. 35, no. 1, pp. 105-112, 2012.

[19] S. A. Black, K. S. Markides, and L. A. Ray, "Depression predicts increased incidence of adverse health outcomes in older Mexican Americans with type 2 diabetes," Diabetes Care, vol. 26, no. 10, pp. 2822-2828, 2003.

[20] E. Atlantis, P. Fahey, and J. Foster, "Collaborative care for comorbid depression and diabetes: a systematic review and meta-analysis," BMJ Open, vol. 4, no. 4, Article ID e004706, 2014.

[21] E. A. Beverly, "Stressing the importance of diabetes distress: a comment on Baek et al," Annals of Behavioral Medicine, vol. 48, no. 2, pp. 137-139, 2014.

[22] D. G. Bruce, W. A. Davis, S. E. Starkstein, and T. M. E. Davis, "A prospective study of depression and mortality in patients with 
type 2 diabetes: the Fremantle Diabetes Study," Diabetologia, vol. 48, no. 12, pp. 2532-2539, 2005.

[23] D. Hessler, L. Fisher, R. E. Glasgow et al., "Reductions in regimen distress are associated with improved management and glycemic control over time," Diabetes Care, vol. 37, no. 3, pp. 617624, 2014.

[24] S. A. Safren, J. S. Gonzalez, D. J. Wexler et al., "A randomized controlled trial of cognitive behavioral therapy for adherence and depression (CBT-AD) in patients with uncontrolled type 2 diabetes," Diabetes Care, vol. 37, no. 3, pp. 625-633, 2014.

[25] L. Fishman and E. Saltonstall, "Yoga in pain management," in Integrative Pain Medicine: The Science and Practice of Complementary and Alternative Medicine in Pain Management, pp. 259-284, Humana Press, 2008.

[26] M. Garfinkel and H. R. Schumacher, "Yoga," Rheumatic Disease Clinics of North America, vol. 26, no. 1, pp. 125-132, 2000.

[27] K. Chandler, "The emerging field of yoga therapy," Hawaii Medical Journal, vol. 60, no. 11, pp. 286-287, 2001.

[28] J. A. Raub, "Psychophysiologic effects of Hatha Yoga on musculoskeletal and cardiopulmonary function: a literature review," Journal of Alternative and Complementary Medicine, vol. 8, no. 6, pp. 797-812, 2002.

[29] C. K. Chappie, "Modern yoga," Religious Studies Review, vol. 34, no. 2, pp. 71-76, 2008

[30] P. E. Jeter, J. Slutsky, N. Singh, and S. B. Khalsa, "Yoga as a therapeutic intervention: a bibliometric analysis of published research studies from 1967 to 2013," The Journal of Alternative and Complementary Medicine, 2015.

[31] A. Ross and S. Thomas, "The health benefits of yoga and exercise: a review of comparison studies," Journal of Alternative and Complementary Medicine, vol. 16, no. 1, pp. 3-12, 2010.

[32] A. Büssing, A. Michalsen, S. B. S. Khalsa, S. Telles, and K. J. Sherman, "Effects of yoga on mental and physical health: a short summary of reviews," Evidence-Based Complementary and Alternative Medicine, vol. 2012, Article ID 165410, 7 pages, 2012.

[33] N. K. Patel, A. H. Newstead, and R. L. Ferrer, "The effects of yoga on physical functioning and health related quality of life in older adults: a systematic review and meta-analysis," Journal of Alternative and Complementary Medicine, vol. 18, no. 10, pp. 902-917, 2012.

[34] H. Cramer, R. Lauche, H. Haller, N. Steckhan, A. Michalsen, and G. Dobos, "Effects of yoga on cardiovascular disease risk factors: a systematic review and meta-analysis," International Journal of Cardiology, vol. 173, no. 2, pp. 170-183, 2014.

[35] L. A. Uebelacker, G. Epstein-Lubow, B. A. Gaudiano, G. Tremont, C. L. Battle, and I. W. Miller, "Hatha yoga for depression: Critical review of the evidence for efficacy, plausible mechanisms of action, and directions for future research," Journal of Psychiatric Practice, vol. 16, no. 1, pp. 22-33, 2010.

[36] M. Balasubramaniam, S. Telles, and P. M. Doraiswamy, "Yoga on our minds: a systematic review of yoga for neuropsychiatric disorders," Frontiers in Psychiatry, vol. 3, article 117, 2013.

[37] H. Cramer, R. Lauche, J. Langhorst, and G. Dobos, "Yoga for depression: a systematic review and meta-analysis," Depression and Anxiety, vol. 30, no. 11, pp. 1068-1083, 2013.

[38] K. Khare and D. Jain, "Effect of yoga on plasma glucose and serum fructosamine level in NIDDM," Yoga Mimamsa, vol. 33, pp. 1-9, 1999.

[39] M. Bindra, S. Nair, and S. Darotiya, "Influence of pranayamas and yoga-asanas on blood glucose, lipid profile and HbAlc in type 2 diabetes," International Journal of Pharma and Bio Sciences, vol. 4, no. 1, pp. 169-172, 2013.
[40] V. V. Agte and K. Tarwadi, "Sudarshan kriya yoga for treating type 2 diabetes: a preliminary study," Alternative and Complementary Therapies, vol. 10, no. 4, pp. 220-222, 2004.

[41] H. H. Mahapure, S. U. Shete, and T. K. Bera, "Effect of yogic exercise on super oxide dismutase levels in diabetics," International Journal of Yoga, vol. 1, no. 1, pp. 21-26, 2008.

[42] S. Dash and A. K. Thakur, "Effect of yoga in patient's with typeII diabetes mellitus," Journal of Evolution of Medical and Dental Sciences, vol. 3, no. 7, pp. 1642-1655, 2014.

[43] U. Popli, C. P. Subbe, and K. Sunil, "Research letter-the role of yoga as a lifestyle modification in treatment of diabetes mellitus: results of a pilot study," Alternative Therapies in Health and Medicine, vol. 20, no. 6, pp. 24-26, 2014.

[44] P. A. Balaji, S. R. Varne, and S. S. Ali, "Effects of yogapranayama practices on metabolic parameters and anthropometry in type 2 diabetes," International Multidisciplinary Research Journal, vol. 1, no. 10, pp. 1-4, 2011.

[45] S. V. Hegde, P. Adhikari, S. Kotian, V. J. Pinto, S. D’Souza, and V. D'Souza, "Effect of 3-month yoga on oxidative stress in type 2 diabetes with or without complications: a controlled clinical trial," Diabetes Care, vol. 34, no. 10, pp. 2208-2210, 2011.

[46] T. Kyizom, S. Singh, K. P. Singh, O. P. Tandon, and R. Kumar, "Effect of pranayama \& yoga-asana on cognitive brain functions in type 2 diabetes-P3 event related evoked potential (ERP)," Indian Journal of Medical Research, vol. 131, no. 5, pp. 636-640, 2010.

[47] V. Malhotra, S. Singh, S. B. Sharma et al., "The status of NIDDM patients after yoga asanas: assessment of important parameters," Journal of Clinical and Diagnostic Research, vol. 4, no. 3, pp. 2652-2667, 2010.

[48] S. Singh, T. Kyizom, K. P. Singh, O. P. Tandon, and S. V. Madhu, "Influence of pranayamas and yoga-asanas on serum insulin, blood glucose and lipid profile in type 2 diabetes," Indian Journal of Clinical Biochemistry, vol. 23, no. 4, pp. 365-368, 2008.

[49] R. Agrawal, R. Aradhana, S. Hussain, R. Beniwal, M. Sabir, and D. Kochar, "Influence of yogic treatment on quality of life outcomes, glycaemic control and risk factors in diabetes mellitus," International Journal of Diabetes in Developing Countries, vol. 23, pp. 130-134, 2003.

[50] V. Malhotra, S. Singh, O. P. Tandon, S. V. Madhu, A. Prasad, and S. B. Sharma, "Effect of Yoga asanas on nerve conduction in type 2 diabetes," Indian Journal of Physiology \& Pharmacology, vol. 46, no. 3, pp. 298-306, 2002.

[51] N. Habibi and S. Marandi, "Effect of 12 weeks of yoga practice on glucose, insulin and triglycerides serum level in women with diabetes type II," Journal of Gorgan University of Medical Sciences, vol. 15, no. 4, pp. 1-7, 2014.

[52] N. Habibi, Z. Farsani, B. Yazdani, R. Arianshakib, and P. Noruozi, "The influence of yoga on risk profiles programs in women with diabetes type II," Advances in Environmental Biology, vol. 7, no. 4, pp. 550-555, 2013.

[53] V. P. Jyotsna, A. Dhawan, V. Sreenivas, K. K. Deepak, and R. Singla, "Completion report: effect of Comprehensive Yogic Breathing program on type 2 diabetes: a randomized control trial," Indian Journal of Endocrinology and Metabolism, vol. 18, no. 4, pp. 582-584, 2014.

[54] V. P. Jyotsna, S. Ambekar, R. Singla et al., "Cardiac autonomic function in patients with diabetes improves with practice of comprehensive yogic breathing program," Indian Journal of Endocrinology and Metabolism, vol. 17, no. 3, pp. 480-485, 2013.

[55] V. P. Jyotsna, S. Ambekar, A. Joshi et al., "Prospective randomized controlled intervention trial: comprehensive yogic 
breathing program improves cardiac autonomic functions and quality of life in diabetes," Indian Journal of Endocrinology and Metabolism, vol. 16, supplement 2, pp. S489-S491, 2012.

[56] V. P. Jyotsna, A. Joshi, S. Ambekar, N. Kumar, A. Dhawan, and V. Sreenivas, "Comprehensive yogic breathing program improves quality of life in patients with diabetes," Indian Journal of Endocrinology and Metabolism, vol. 16, no. 3, pp. 423-428, 2012.

[57] R. Nagarathna, M. R. Usharani, A. R. Rao, R. Chaku, R. Kulkarni, and H. R. Nagendra, "Efficacy of yoga based life style modification program on medication score and lipid profile in type 2 diabetes-a randomized control study," International Journal of Diabetes in Developing Countries, vol. 32, no. 3, pp. 122-130, 2012.

[58] N. Shantakumari, S. Sequeira, and R. El Deeb, "Effects of a yoga intervention on lipid profiles of diabetes patients with dyslipidemia," Indian Heart Journal, vol. 65, no. 2, pp. 127-131, 2013.

[59] N. Shantakumari, S. Sequeira, and R. Eldeeb, "Effect of a yoga intervention on hypertensive diabetic patients," Journal of Advances in Internal Medicine, vol. 1, no. 2, pp. 60-63, 2012.

[60] T. G. Subramaniyan, N. Subramaniyan, and M. Chidambaram, "Brisk walking and yoga as adjuvant therapy in management of type 2 diabetes mellitus," International Journal of Students Research, vol. 2, no. 1, pp. 43-46, 2012.

[61] K. Vaishali, K. V. Kumar, P. Adhikari, and B. UnniKrishnan, "Effects of yoga-based program on glycosylated hemoglobin level serum lipid profile in community dwelling elderly subjects with chronic type 2 diabetes mellitus-a randomized controlled trial," Physical \& Occupational Therapy in Geriatrics, vol. 30, no. 1, pp. 22-30, 2012.

[62] K. Vaishali, K. V. Kumar, P. Adhikari, and B. Unni Krishnan, "Effects of yoga-based program on glycosylated hemoglobin level and serum lipid profile in community dwelling elderly subjects with chronic type 2 diabetes mellitus-a randomized controlled trial," Indian Journal of Ancient Medicine and Yoga, vol. 4, no. 2, pp. 69-76, 2011.

[63] A. Pardasany, S. Shenoy, and J. S. Sandhu, "Comparing the efficacy of tai chi chuan and hatha yoga in type 2 diabetes mellitus patients on parameters of blood glucose control and lipid metabolism," Indian Journal of Physiotherapy and Occupational Therapy, vol. 4, no. 3, pp. 11-16, 2010.

[64] S. Amita, S. Prabhakar, I. Manoj, S. Harminder, and T. Pavan, "Effect of Yoga-Nidra on blood glucose level in diabetic patients," Indian Journal of Physiology and Pharmacology, vol. 53, no. 1, pp. 97-101, 2009.

[65] L. Skoro-Kondza, S. S. Tai, R. Gadelrab, D. Drincevic, and T. Greenhalgh, "Community based yoga classes for type 2 diabetes: an exploratory randomised controlled trial," $B M C$ Health Services Research, vol. 9, article 33, 2009.

[66] L. A. Gordon, E. Y. Morrison, D. A. McGrowder et al., "Effect of exercise therapy on lipid profile and oxidative stress indicators in patients with type 2 diabetes," BMC Complementary and Alternative Medicine, vol. 8, article 21, 2008.

[67] L. Gordon, E. Y. Morrison, D. A. McGrowder et al., "Changes in clinical and metabolic parameters after exercise therapy in patents with type 2 diabetes," Archives of Medical Science, vol. 4, no. 4, pp. 427-437, 2008.

[68] L. Gordon, E. Y. Morrison, D. McGrowder et al., "Effect of yoga and traditional physical exercise on hormones and percentage insulin binding receptor in patients with type 2 diabetes,"
American Journal of Biochemistry and Biotechnology, vol. 4, no. 1, pp. 35-42, 2008.

[69] E. M. Céspedes, G. Riverón, C. A. Alonso, and L. Gordon, "Evolución metabólica de pacientes diabéticos tipo 2 sometidos a un tratamiento combinado de dieta y ejercicios yoga," Revista Cubana de Investigaciones Biomedicas, vol. 21, no. 2, pp. 1-5, 2002.

[70] R. Monro, J. Power, A. Coumar, R. Nagarathna, and P. Dandona, "Yoga therapy for NIDDM: a controlled trial," Complementary Medical Research, vol. 6, no. 2, pp. 66-68, 1992.

[71] P. Chu, R. A. Gotink, G. Y. Yeh, S. J. Goldie, and M. G. M. Hunink, "The effectiveness of yoga in modifying risk factors for cardiovascular disease and metabolic syndrome: a systematic review and meta-analysis of randomized controlled trials," European Journal of Preventive Cardiology, 2014.

[72] H. Cramer, H. Haller, R. Lauche, N. Steckhan, A. Michalsen, and G. Dobos, "A systematic review and meta-analysis of yoga for hypertension," American Journal of Hypertension, vol. 27, no. 9, pp. 1146-1151, 2014.

[73] M. Hagins, R. States, T. Selfe, and K. Innes, "Effectiveness of yoga for hypertension: systematic review and meta-analysis," Evidence-Based Complementary and Alternative Medicine, vol. 2013, Article ID 649836, 13 pages, 2013.

[74] A. Ceriello, "Oxidative stress and diabetes-associated complications," Endocrine Practice, vol. 12, supplement 1, pp. 60-62, 2006.

[75] H. K. Vincent, K. E. Innes, and K. R. Vincent, "Oxidative stress and potential interventions to reduce oxidative stress in overweight and obesity," Diabetes, Obesity and Metabolism, vol. 9, no. 6, pp. 813-839, 2007.

[76] K. E. Innes, H. K. Vincent, and A. G. Taylor, "Chronic stress and insulin-resistance-related indices of cardiovascular disease risk. Part I. Neurophysiological responses and pathological sequelae," Alternative Therapies in Health and Medicine, vol. 13, no. 4, pp. 46-52, 2007.

[77] A. C. Maritim, R. A. Sanders, and J. B. Watkins III, "Diabetes, oxidative stress, and antioxidants: a review," Journal of Biochemical \& Molecular Toxicology, vol. 17, no. 1, pp. 24-38, 2003.

[78] K. Stadler, "Oxidative stress in diabetes," in Diabetes Advances in Experimental Medicine and Biology, S. I. Ahmad, Ed., pp. 272287, Landes Bioscience and Springer Science, New York, NY, USA, 2013.

[79] A. Nouwen, K. Winkley, J. Twisk et al., “Type 2 diabetes mellitus as a risk factor for the onset of depression: a systematic review and meta-analysis," Diabetologia, vol. 53, no. 12, pp. 2480-2486, 2010.

[80] K. E. Innes, T. K. Selfe, and A. G. Taylor, "Menopause, the metabolic syndrome, and mind-body therapies," Menopause, vol. 15, no. 5, pp. 1005-1013, 2008.

[81] K. E. Innes, H. K. Vincent, and A. G. Taylor, "Chronic stress and insulin resistance-related indices of cardiovascular disease risk, part I: neurophysiological responses and pathological sequelae," Alternative Therapies in Health and Medicine, vol. 13, no. 4, pp. 46-52, 2007.

[82] A. A. Weinstein, P. A. Deuster, and W. J. Kop, "Heart rate variability as a predictor of negative mood symptoms induced by exercise withdrawal," Medicine \& Science in Sports \& Exercise, vol. 39, no. 4, pp. 735-741, 2007.

[83] S. H. Golden, "A review of the evidence for a neuroendocrine link between stress, depression and diabetes mellitus," Current Diabetes Reviews, vol. 3, no. 4, pp. 252-259, 2007. 
[84] I. Kyrou, G. P. Chrousos, and C. Tsigos, "Stress, visceral obesity, and metabolic complications," Annals of the New York Academy of Sciences, vol. 1083, pp. 77-110, 2006.

[85] L. P. Reagan, C. A. Grillo, and G. G. Piroli, "The As and Ds of stress: metabolic, morphological and behavioral consequences," European Journal of Pharmacology, vol. 585, no. 1, pp. 64-75, 2008.

[86] B. T. Mausbach, S. Ancoli-Israel, R. von Känel et al., "Sleep disturbance, norepinephrine, and D-dimer are all related in elderly caregivers of people with Alzheimer disease," Sleep, vol. 29, no. 10, pp. 1347-1352, 2006.

[87] B. S. McEwen, "Sleep deprivation as a neurobiologic and physiologic stressor: allostasis and allostatic load," Metabolism, vol. 55, no. 10, supplement 2, pp. S20-S23, 2006.

[88] B. S. McEwen, "Central effects of stress hormones in health and disease: understanding the protective and damaging effects of stress and stress mediators," European Journal of Pharmacology, vol. 583, no. 2-3, pp. 174-185, 2008.

[89] P. H. Whincup, S. J. Kaye, C. G. Owen et al., "Birth weight and risk of type 2 diabetes: a systematic review," The Journal of the American Medical Association, vol. 300, no. 24, pp. 2886-2897, 2008.

[90] M. J. Knol, J. W. Twisk, A. T. Beekman, R. J. Heine, F. J. Snoek, and F. Pouwer, "Depression as a risk factor for the onset of type 2 diabetes mellitus. A meta-analysis," Diabetologia, vol. 49, no. 5, pp. 837-845, 2006.

[91] A.-K. Eriksson, A. Ekbom, F. Granath, A. Hilding, S. Efendic, and C.-G. Östenson, "Psychological distress and risk of prediabetes and Type 2 diabetes in a prospective study of Swedish middle-aged men and women," Diabetic Medicine, vol. 25, no. 7, pp. 834-842, 2008.

[92] A. Engum, "The role of depression and anxiety in onset of diabetes in a large population-based study," Journal of Psychosomatic Research, vol. 62, no. 1, pp. 31-38, 2007.

[93] E. C. Suarez, "Self-reported symptoms of sleep disturbance and inflammation, coagulation, insulin resistance and psychosocial distress: evidence for gender disparity, Brain, Behavior, and Immunity, vol. 22, no. 6, pp. 960-968, 2008.

[94] M. I. Trenell, N. S. Marshall, and N. L. Rogers, "Sleep and metabolic control: waking to a problem?" Clinical \& Experimental Pharmacology \& Physiology, vol. 34, no. 1-2, pp. 1-9, 2007.

[95] M. R. Irwin, M. Wang, D. Ribeiro et al., "Sleep loss activates cellular inflammatory signaling," Biological Psychiatry, vol. 64, no. 6, pp. 538-540, 2008.

[96] C. Meisinger, M. Heier, and H. Loewel, "Sleep disturbance as a predictor of type 2 diabetes mellitus in men and women from the general population," Diabetologia, vol. 48, no. 2, pp. 235-241, 2005.

[97] P. M. Nilsson, M. Rööst, G. Engström, B. Hedblad, and G. Berglund, "Incidence of diabetes in middle-aged men is related to sleep disturbances," Diabetes Care, vol. 27, no. 10, pp. 24642469, 2004.

[98] M. J. Knol, J. W. R. Twisk, A. T. F. Beekman, R. J. Heine, F. J. Snoek, and F. Pouwer, "Depression as a risk factor for the onset of type 2 diabetes mellitus. A meta-analysis," Diabetologia, vol. 49, no. 5, pp. 837-845, 2006.

[99] O. Chan, K. Inouye, M. C. Riddell, M. Vranic, and S. G. Matthews, "Diabetes and the hypothalamo-pituitary-adrenal (HPA) axis," Minerva Endocrinologica, vol. 28, no. 2, pp. 87-102, 2003.
[100] R. S. Surwit, M. A. L. van Tilburg, N. Zucker et al., "Stress management improves long-term glycemic control in type 2 diabetes," Diabetes Care, vol. 25, no. 1, pp. 30-34, 2002.

[101] E. B. Fisher, C. T. Thorpe, B. M. Devellis, and R. F. Devellis, "Healthy coping, negative emotions, and diabetes management: a systematic review and appraisal," Diabetes Educator, vol. 33, no. 6, pp. 1080-1103, 2007.

[102] P. A. Pirraglia and S. Gupta, "The interaction of depression and diabetes: a review," Current Diabetes Reviews, vol. 3, no. 4, pp. 249-251, 2007.

[103] J. C. Konen, J. H. Summerson, and M. B. Dignan, "Family function, stress, and locus of control. Relationships to glycemia in adults with diabetes mellitus," Archives of Family Medicine, vol. 2, no. 4, pp. 393-402, 1993.

[104] K. L. Knutson, E. Van Cauter, P. Zee, K. Liu, and D. S. Lauderdale, "Cross-sectional associations between measures of sleep and markers of glucose metabolism among subjects with and without diabetes. The coronary artery risk development in young adults (CARDIA) sleep study," Diabetes Care, vol. 34, no. 5, pp. 1171-1176, 2011.

[105] F. P. Cappuccio, L. D’Elia, P. Strazzullo, and M. A. Miller, "Sleep duration and all-cause mortality: a systematic review and metaanalysis of prospective studies," Sleep, vol. 33, no. 5, pp. 585-592, 2010.

[106] X. Zhang, S. L. Norris, E. W. Gregg, Y. J. Cheng, G. Beckles, and H. S. Kahn, "Depressive symptoms and mortality among persons with and without diabetes," American Journal of Epidemiology, vol. 161, no. 7, pp. 652-660, 2005.

[107] W. J. Katon, C. Rutter, G. Simon et al., "The association of comorbid depression with mortality in patients with type 2 diabetes," Diabetes Care, vol. 28, no. 11, pp. 2668-2672, 2005.

[108] E. H. B. Lin, S. R. Heckbert, C. M. Rutter et al., "Depression and increased mortality in diabetes: unexpected causes of death," Annals of Family Medicine, vol. 7, no. 5, pp. 414-421, 2009.

[109] W. Katon, M.-Y. Fan, J. Unützer, J. Taylor, H. Pincus, and M. Schoenbaum, "Depression and diabetes: a potentially lethal combination," Journal of General Internal Medicine, vol. 23, no. 10, pp. 1571-1575, 2008.

[110] K.-M. Chen, M.-H. Chen, H.-C. Chao, H.-M. Hung, H.-S. Lin, and C.-H. Li, "Sleep quality, depression state, and health status of older adults after silver yoga exercises: cluster randomized trial," International Journal of Nursing Studies, vol. 46, no. 2, pp. 154-163, 2009.

[111] K.-M. Chen, M.-H. Chen, M.-H. Lin, J.-T. Fan, H.-S. Lin, and C.-H. Li, "Effects of yoga on sleep quality and depression in elders in assisted living facilities," The Journal of Nursing Research, vol. 18, no. 1, pp. 53-61, 2010.

[112] K.-Y. Lin, Y.-T. Hu, K.-J. Chang, H.-F. Lin, and J.-Y. Tsauo, "Effects of yoga on psychological health, quality of life, and physical health of patients with cancer: a meta-analysis," Evidence-Based Complementary and Alternative Medicine, vol. 2011, Article ID 659876, 12 pages, 2011.

[113] K. E. Innes, H. K. Vincent, and A. G. Taylor, "Chronic stress and insulin resistance-related indices of cardiovascular disease risk, part 2: a potential role for mind-body therapies," Alternative Therapies in Health and Medicine, vol. 13, no. 5, pp. 44-51, 2007, Erratum in: Alternative Therapies in Health and Medicine, vol. 13, no. 6, p. 15, 2007.

[114] C. C. Streeter, Theodore H. Whitfield, L. Owen et al., "Effects of yoga versus walking on mood, anxiety, and brain GABA levels: a randomized controlled MRS study," Journal of Alternative and Complementary Medicine, vol. 16, no. 11, pp. 1145-1152, 2010. 
[115] G. Kirkwood, H. Rampes, V. Tuffrey, J. Richardson, and K. Pilkington, "Yoga for anxiety: a systematic review of the research evidence," British Journal of Sports Medicine, vol. 39, no. 12, pp. 884-891, 2005.

[116] K. E. Innes, T. K. Selfe, P. Agarwal, K. Williams, and K. L. Flack, "Efficacy of an eight-week yoga intervention on symptoms of restless legs syndrome (RLS): a pilot study," Journal of Alternative and Complementary Medicine, vol. 19, no. 6, pp. 527535, 2013.

[117] K. E. Innes and T. K. Selfe, "The effects of a gentle yoga program on sleep, mood, and blood pressure in older women with restless legs syndrome (RLS): a preliminary randomized controlled trial," Evidence-Based Complementary and Alternative Medicine, vol. 2012, Article ID 294058, 14 pages, 2012.

[118] K. E. Innes, H. K. Vincent, and A. G. Taylor, "Chronic stress and insulin resistance-related indices of cardiovascular disease risk. Part 2. A potential role for mind-body therapies," Alternative Therapies in Health and Medicine, vol. 13, no. 5, pp. 44-51, 2007.

[119] K. E. Innes and H. K. Vincent, "The influence of yogabased programs on risk profiles in adults with type 2 diabetes mellitus: a systematic review," Evidence-Based Complementary and Alternative Medicine, vol. 4, no. 4, pp. 469-486, 2007.

[120] M. R. Carnethon, R. J. Prineas, M. Temprosa, Z.-M. Zhang, G. Uwaifo, and M. E. Molitch, "The association among autonomic nervous system function, incident diabetes, and intervention arm in the diabetes prevention program," Diabetes Care, vol. 29, no. 4, pp. 914-919, 2006.

[121] M. R. Carnethon, S. H. Golden, A. R. Folsom, W. Haskell, and D. Liao, "Prospective investigation of autonomic nervous system function and the development of type 2 diabetes: the atherosclerosis risk in communities study, 1987-1998," Circulation, vol. 107, no. 17, pp. 2190-2195, 2003.

[122] R. B. Singh, C. Kartik, K. Otsuka, D. Pella, and J. Pella, "Brainheart connection and the risk of heart attack," Biomedicine and Pharmacotherapy, vol. 56, supplement 2, pp. 257s-265s, 2002.

[123] R. E. Maser, B. D. Mitchell, A. I. Vinik, and R. Freeman, “The association between cardiovascular autonomic neuropathy and mortality in individuals with diabetes: a meta-analysis," Diabetes Care, vol. 26, no. 6, pp. 1895-1901, 2003.

[124] D. Manzella and G. Paolisso, "Cardiac autonomic activity and type II diabetes mellitus," Clinical Science, vol. 108, no. 2, pp. 9399, 2005.

[125] K. E. Innes, C. Bourguignon, and A. G. Taylor, "Risk indices associated with the insulin resistance syndrome, cardiovascular disease, and possible protection with yoga: a systematic review," Journal of the American Board of Family Practice, vol. 18, no. 6, pp. 491-519, 2005.

[126] K. Innes, "Yoga-based programs for cardiovascular disease, diabetes, and related chronic conditions. Time for a new health care paradigm?" in Proceedings of the Yoga and Health: Research and Practice-An International Conference, London, UK, April 2014.

[127] S. Tiwari, S. K. Tiwari, S. Gehlot, and G. Singh, "Outcome of breathing exercise (pranayam) on spirometric parameters in type 2 diabetic individuals: a clinical study," Journal of Stress Physiology \& Biochemistry, vol. 8, no. 4, pp. 218-225, 2012.

[128] D. A. Lawlor, S. Ebrahim, and G. D. Smith, "Associations of measures of lung function with insulin resistance and Type 2 diabetes: findings from the British Women's Heart and Health study," Diabetologia, vol. 47, no. 2, pp. 195-203, 2004.

[129] W. A. Davis, M. Knuiman, P. Kendall, V. Grange, and T. M. E. Davis, "Glycemic exposure is associated with reduced pulmonary function in type 2 diabetes: the Fremantle Diabetes Study," Diabetes Care, vol. 27, no. 3, pp. 752-757, 2004.

[130] E. S. Ford, D. M. Mannino, and National Health and Nutrition Examination Survey Epidemiologic Follow-Up Study, "Prospective association between lung function and the incidence of diabetes: findings from the National Health and Nutrition Examination Survey Epidemiologic Follow-up Study," Diabetes Care, vol. 27, no. 12, pp. 2966-2970, 2004.

[131] T. M. McKeever, P. J. Weston, R. Hubbard, and A. Fogarty, "Lung function and glucose metabolism: an analysis of data from the Third National Health and Nutrition Examination Survey," American Journal of Epidemiology, vol. 161, no. 6, pp. 546-556, 2005.

[132] E. de G R Hansen and K. E. Innes, “The benefits of yoga for adults with type 2 diabetes: a review of the evidence and call for a collaborative, integrated research initiative," International Journal of Yoga Therapy, vol. 23, no. 2, pp. 71-83, 2013.

[133] K. E. Innes, C. Bourguignon, and A. G. Taylor, "Risk indices associated with the insulin resistance syndrome, cardiovascular disease, and possible protection with yoga: a systematic review," The Journal of the American Board of Family Medicine, vol. 18, no. 6, pp. 491-519, 2005.

[134] D. Khatri, K. C. Mathur, S. Gahlot, S. Jain, and R. P. Agrawal, "Effects of yoga and meditation on clinical and biochemical parameters of metabolic syndrome," Diabetes Research and Clinical Practice, vol. 78, no. 3, pp. e9-e10, 2007.

[135] I. Kyrou and C. Tsigos, "Stress mechanisms and metabolic complications," Hormone \& Metabolic Research, vol. 39, no. 6, pp. 430-438, 2007.

[136] I. Kyrou, G. P. Chrousos, and C. Tsigos, "Stress, visceral obesity, and metabolic complications," Stress, Obesity, and Metabolic Syndrome, vol. 1083, pp. 77-110, 2006.

[137] R. P. Brown and P. L. Gerbarg, "Sudarshan Kriya yogic breathing in the treatment of stress, anxiety, and depression: part Ineurophysiologic model," The Journal of Alternative and Complementary Medicine, vol. 11, no. 1, pp. 189-201, 2005, Erratum in: The Journal of Alternative and Complementary Medicine, vol. 11, no. 2, pp. 383-384, 2005.

[138] R. McCaffrey, P. Ruknui, U. Hatthakit, and P. Kasetsomboon, "The effects of yoga on hypertensive persons in Thailand," Holistic Nursing Practice, vol. 19, no. 4, pp. 173-180, 2005.

[139] K. Khattab, A. A. Khattab, J. Ortak, G. Richardt, and H. Bonnemeier, "Iyengar yoga increases cardiac parasympathetic nervous modulation among healthy yoga practitioners," EvidenceBased Complementary and Alternative Medicine, vol. 4, no. 4, pp. 511-517, 2007.

[140] R. E. De Meersman and P. K. Stein, "Vagal modulation and aging," Biological Psychology, vol. 74, no. 2, pp. 165-173, 2007.

[141] J. F. Thayer and E. Sternberg, "Beyond heart rate variability," Annals of the New York Academy of Sciences, vol. 1088, pp. 361372, 2006.

[142] J. F. Thayer and R. D. Lane, "The role of vagal function in the risk for cardiovascular disease and mortality," Biological Psychology, vol. 74, no. 2, pp. 224-242, 2007.

[143] D. J. J. Wang, H. Rao, M. Korczykowski et al., "Cerebral blood flow changes associated with different meditation practices and perceived depth of meditation," Psychiatry Research: Neuroimaging, vol. 191, no. 1, pp. 60-67, 2011.

[144] A. B. Newberg, N. Wintering, M. R. Waldman, D. Amen, D. S. Khalsa, and A. Alavi, "Cerebral blood flow differences between long-term meditators and non-meditators," Consciousness and Cognition, vol. 19, no. 4, pp. 899-905, 2010. 
[145] K. Rubia, "The neurobiology of meditation and its clinical effectiveness in psychiatric disorders," Biological Psychology, vol. 82, no. 1, pp. 1-11, 2009.

[146] T. W. Kjaer, C. Bertelsen, P. Piccini, D. Brooks, J. Alving, and H. C. Lou, "Increased dopamine tone during meditation-induced change of consciousness," Cognitive Brain Research, vol. 13, no. 2, pp. 255-259, 2002.

[147] G. K. Alexander, K. E. Innes, T. K. Selfe, and C. J. Brown, “"More than I expected': perceived benefits of yoga practice among older adults at risk for cardiovascular disease," Complementary Therapies in Medicine, vol. 21, no. 1, pp. 14-28, 2013.

[148] M. A. Steinhardt, M. M. Mamerow, S. A. Brown, and C. A. Jolly, "A resilience intervention in African American adults with type 2 diabetes: a pilot study of efficacy," Diabetes Educator, vol. 35, no. 2, pp. 274-284, 2009.

[149] J. P. Yi, P. P. Vitaliano, R. E. Smith, J. C. Yi, and K. Weinger, "The role of resilience on psychological adjustment and physical health in patients with diabetes," British Journal of Health Psychology, vol. 13, no. 2, pp. 311-325, 2008.

[150] N. Hartfiel, J. Havenhand, S. B. Khalsa, G. Clarke, and A. Krayer, "The effectiveness of yoga for the improvement of well-being and resilience to stress in the workplace," Scandinavian Journal of Work, Environment \& Health, vol. 37, no. 1, pp. 70-76, 2011.

[151] J. Johnston, "The impact of yoga on military personnel with post traumatic stress disorder," Paper 29, Northeastern University, Boston, Mass, USA, 2011.

[152] J. J. Noggle, N. J. Steiner, T. Minami, and S. B. S. Khalsa, "Benefits of yoga for psychosocial well-being in a us high school curriculum: a preliminary randomized controlled trial," Journal of Developmental and Behavioral Pediatrics, vol. 33, no. 3, pp. 193-201, 2012.

[153] J. Dalen, B. W. Smith, B. M. Shelley, A. L. Sloan, L. Leahigh, and D. Begay, "Pilot study: mindful Eating and Living (MEAL): weight, eating behavior, and psychological outcomes associated with a mindfulness-based intervention for people with obesity," Complementary Therapies in Medicine, vol. 18, no. 6, pp. 260264, 2010.

[154] K. A. Dittmann and M. R. Freedman, "Body awareness, eating attitudes, and spiritual beliefs of women practicing Yoga," Eating Disorders, vol. 17, no. 4, pp. 273-292, 2009.

[155] H. A. van Dam, F. G. van der Horst, L. Knoops, R. M. Ryckman, H. F. J. M. Crebolder, and B. H. W. van den Borne, "Social support in diabetes: a systematic review of controlled intervention studies," Patient Education and Counseling, vol. 59, no. 1, pp. 1-12, 2005.

[156] T. S. Tang, M. B. Brown, M. M. Funnell, and R. M. Anderson, "Social support, quality of life, and self-care behaviors among african americans with type 2 diabetes," Diabetes Educator, vol. 34, no. 2, pp. 266-276, 2008.

[157] L. Bernardi, M. Rosengård-Bärlund, and P.-H. Groop, "Cardiorespiratory interactions in diabetes," in Proceedings of the 8th Conference of the European Study Group on Cardiovascular Oscillations (ESGCO '14), pp. 227-228, IEEE, Trento, Italy, May 2014.

[158] L. Bernardi, G. Spadacini, J. Bellwon, R. Hajric, H. Roskamm, and A. W. Frey, "Effect of breathing rate on oxygen saturation and exercise performance in chronic heart failure," The Lancet, vol. 351, no. 9112, pp. 1308-1311, 1998.

[159] L. Pomidori, F. Campigotto, T. M. Amatya, L. Bernardi, and A. Cogo, "Efficacy and tolerability of yoga breathing in patients with chronic obstructive pulmonary disease: a pilot study,"
Journal of Cardiopulmonary Rehabilitation and Prevention, vol. 29, no. 2, pp. 133-137, 2009.

[160] S. Cazzato, F. Bernardi, S. Salardi et al., "Lung function in children with diabetes mellitus," Pediatric Pulmonology, vol. 37, no. 1, pp. 17-23, 2004.

[161] D. S. Black, S. W. Cole, M. R. Irwin et al., "Yogic meditation reverses NF- $\kappa \mathrm{B}$ and IRF-related transcriptome dynamics in leukocytes of family dementia caregivers in a randomized controlled trial," Psychoneuroendocrinology, vol. 38, no. 3, pp. 348-355, 2013.

[162] H. Lavretsky, E. S. Epel, P. Siddarth et al., "A pilot study of yogic meditation for family dementia caregivers with depressive symptoms: effects on mental health, cognition, and telomerase activity," International Journal of Geriatric Psychiatry, vol. 28, no. 1, pp. 57-65, 2013.

[163] J. A. Dusek, H. H. Otu, A. L. Wohlhueter et al., "Genomic counter-stress changes induced by the relaxation response," PLoS ONE, vol. 3, no. 7, Article ID e2576, 2008.

[164] P. Kaliman, M. J. Álvarez-López, M. Cosín-Tomás, M. A. Rosenkranz, A. Lutz, and R. J. Davidson, "Rapid changes in histone deacetylases and inflammatory gene expression in expert meditators," Psychoneuroendocrinology, vol. 40, no. 1, pp. 96-107, 2014.

[165] B. B. Lowell and G. I. Shulman, "Mitochondrial dysfunction and type 2 diabetes," Science, vol. 307, no. 5708, pp. 384-387, 2005.

[166] K. E. Wellen and G. S. Hotamisligil, "Inflammation, stress, and diabetes," The Journal of Clinical Investigation, vol. 115, no. 5, pp. 1111-1119, 2005. 


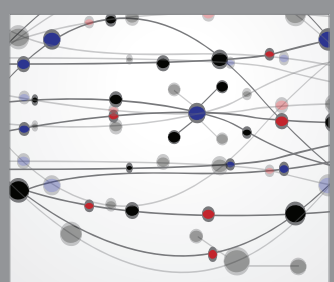

The Scientific World Journal
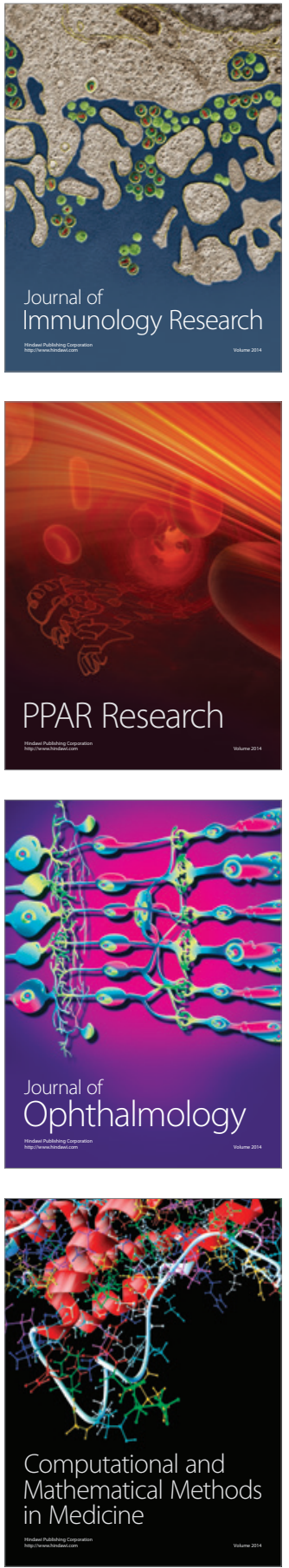

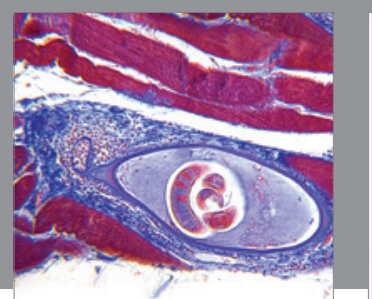

Gastroenterology Research and Practice

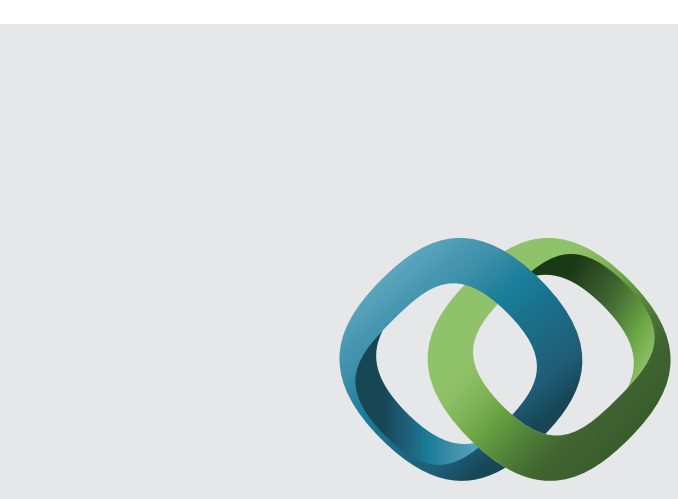

\section{Hindawi}

Submit your manuscripts at

http://www.hindawi.com
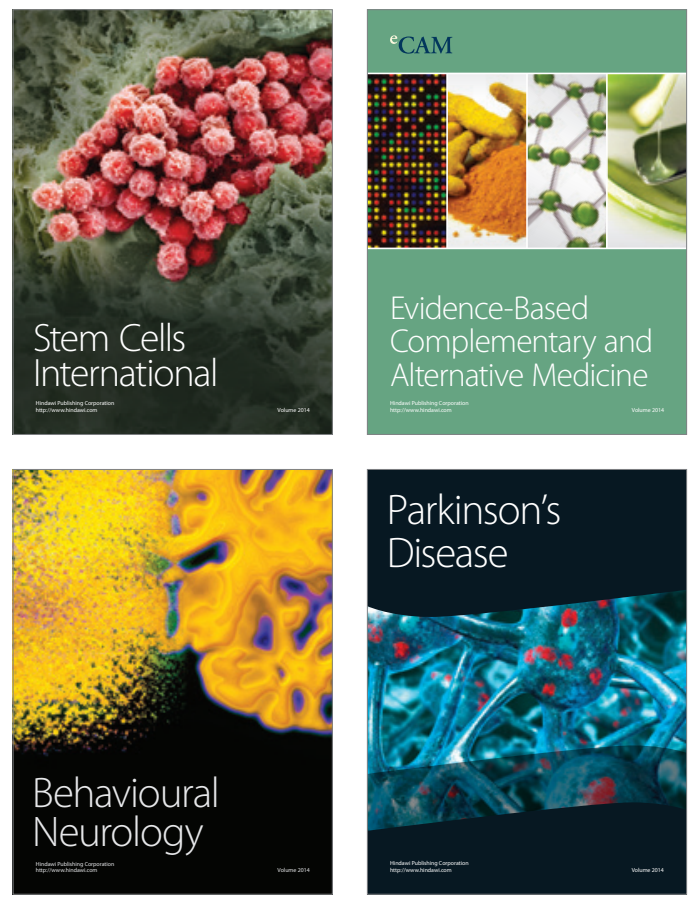
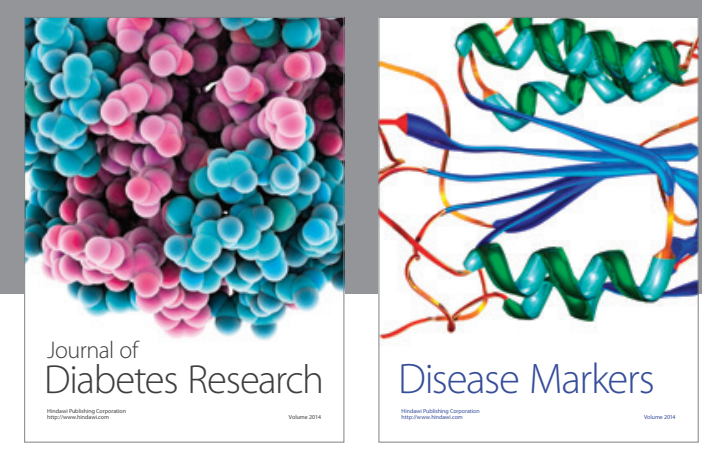

Disease Markers
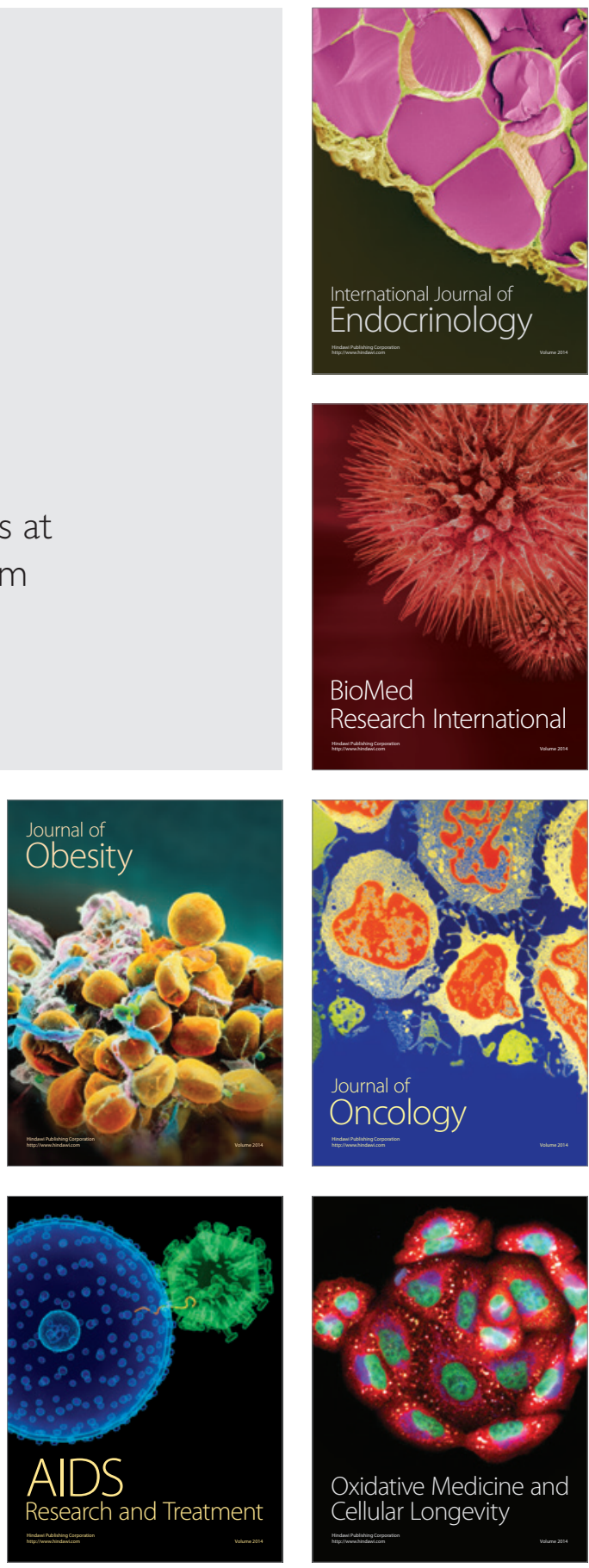